\title{
Supplementary Material:
}

1. Cartesian coordinates (geometries) for $\mathrm{H}_{2} \mathrm{O}$ molecule, used in the generation of EFP (units $\AA$ ) - Monomer $\mathrm{H}_{2} \mathrm{O}$ geometry:

$\begin{array}{lllll}\mathrm{O} & 8.0 & 1.05666840 & -4.08767271 & 3.58133602 \\ \mathrm{H} & 1.0 & 1.73754013 & -3.84632921 & 2.97384501 \\ \mathrm{H} & 1.0 & 1.17789316 & -3.55433559 & 4.35057878\end{array}$

2. Cartesian coordinates (geometries) for $\mathrm{MeOH}$ molecule, used in the generation of EFP (units $\AA$ ) Monomer MeOH geometry:

$\begin{array}{lllll}\mathrm{O} & 8.0 & 0.50935709 & -6.47537136 & 4.37838507 \\ \mathrm{H} & 1.0 & 0.71907318 & -5.58328772 & 4.15927505 \\ \mathrm{C} & 6.0 & 1.18975854 & -7.36236000 & 3.53351521 \\ \mathrm{H} & 1.0 & 0.91052282 & -8.36331367 & 3.83187985 \\ \mathrm{H} & 1.0 & 0.91093189 & -7.22138023 & 2.49224997 \\ \mathrm{H} & 1.0 & 2.26812482 & -7.26160765 & 3.62747478\end{array}$

3. MP2 and EFP2 optimized geometries for three $n=2$ isomers:

EFP2 geometry for $n=2$ (Angstroms):

Isomer 1:

$\begin{array}{lccc}\mathrm{O} & 4.56309700 & -3.18226552 & 4.44334221 \\ \mathrm{H} & 3.90004897 & -3.19248796 & 5.11271954 \\ \mathrm{C} & 5.38183641 & -2.05323672 & 4.57921076 \\ \mathrm{H} & 6.11792231 & -2.09705520 & 3.78854060 \\ \mathrm{H} & 4.81667757 & -1.13043690 & 4.47485828 \\ \mathrm{H} & 5.90057230 & -2.04397058 & 5.53455353 \\ \mathrm{O} & 2.12953949 & -3.01614189 & 6.26952362 \\ \mathrm{H} & 1.46447182 & -2.98205805 & 5.60064268 \\ \mathrm{H} & 1.88246107 & -3.70743370 & 6.86277437 \\ \mathrm{O} & 0.37043759 & -2.77703476 & 3.84743381 \\ \mathrm{H} & 1.01034939 & -2.98821664 & 3.18886113 \\ \mathrm{C} & -0.21169002 & -1.52962399 & 3.58552933 \\ \mathrm{H} & -0.93237156 & -1.34393418 & 4.36977386 \\ \mathrm{H} & 0.52523983 & -0.73046029 & 3.59670210 \\ \mathrm{H} & -0.72999328 & -1.51921344 & 2.62996316 \\ \mathrm{O} & 2.76764727 & -3.29608393 & 2.03876615 \\ \mathrm{H} & 2.85968041 & -4.08021164 & 1.52151179 \\ \mathrm{H} & 3.43168068 & -3.33339739 & 2.70850182 \\ \mathrm{Isomer} & 2 & & \\ \mathrm{O} & 5.39831591 & -0.95749080 & 5.71161747 \\ \mathrm{H} & 5.11720419 & -0.67658275 & 4.85729313 \\ \mathrm{C} & 4.64687967 & -0.32324421 & 6.70990372 \\ \mathrm{H} & 5.01324177 & -0.68357718 & 7.66113472 \\ \mathrm{H} & 4.76668119 & 0.75684589 & 6.67980385 \\ \mathrm{H} & 3.58952856 & -0.56392419 & 6.63286209 \\ \mathrm{O} & 3.76349902 & -3.42198634 & 2.61612439 \\ \mathrm{H} & 4.12364531 & -3.71346545 & 3.43844604 \\ \mathrm{H} & 2.88388109 & -3.76110768 & 2.56978273 \\ \mathrm{O} & 5.06080437 & -3.91303587 & 5.29917526 \\ \mathrm{H} & 5.26328611 & -3.02056217 & 5.53019571 \\ \mathrm{H} & 5.87273979 & -4.39308500 & 5.33370495 \\ \mathrm{O} & 4.33857393 & -0.44314244 & 2.91630864 \\ \mathrm{H} & 4.06656981 & -1.32313085 & 2.71772099 \\ \mathrm{C} & 5.13668871 & 0.06956527 & 1.88497746 \\ \mathrm{H} & 5.41581392 & 1.07484841 & 2.16852117 \\ \mathrm{H} & 6.04277134 & -0.51524466 & 1.74763024 \\ \mathrm{H} & 4.59777355 & 0.11324830 & 0.94183612\end{array}$

Isomer 3: 


$\begin{array}{rrrr}\mathrm{O} & 3.39063430 & -0.73337591 & 6.02489948 \\ \mathrm{H} & 3.04655933 & -0.88868064 & 5.16159439 \\ \mathrm{C} & 4.64171696 & -0.10668742 & 5.95037603 \\ \mathrm{H} & 4.98286629 & 0.04423354 & 6.96513844 \\ \mathrm{H} & 5.37037420 & -0.72188425 & 5.42841387 \\ \mathrm{H} & 4.57929039 & 0.86101812 & 5.45894766 \\ \mathrm{O} & 3.04008865 & -4.52126217 & 4.10239601 \\ \mathrm{H} & 3.15398455 & -4.39157438 & 5.03034353 \\ \mathrm{H} & 2.36384249 & -5.17177105 & 4.00037813 \\ \mathrm{O} & 3.47109175 & -3.58394790 & 6.95798445 \\ \mathrm{H} & 3.40669346 & -2.65441179 & 6.80734015 \\ \mathrm{H} & 2.91170931 & -3.77908397 & 7.69275808 \\ \mathrm{O} & 2.71070313 & -1.62908375 & 3.19391823 \\ \mathrm{H} & 2.73983884 & -2.55710101 & 3.35434985 \\ \mathrm{C} & 3.60191321 & -1.27853489 & 2.17100072 \\ \mathrm{H} & 3.52375126 & -0.20898876 & 2.03365493 \\ \mathrm{H} & 4.62950611 & -1.51962435 & 2.43134999 \\ \mathrm{H} & 3.35104513 & -1.76697409 & 1.23273349\end{array}$

MP2 geometry for $n=2$ (Angstroms):

Isomer 1:

$\begin{array}{lrll}\mathrm{O} & 4.3809108115 & -3.1568086014 & 4.4013701246 \\ \mathrm{H} & 3.7203710467 & -3.1512078439 & 5.0732771488 \\ \mathrm{C} & 5.2861490827 & -2.1044706460 & 4.5927572122 \\ \mathrm{H} & 6.0150231065 & -2.1630312316 & 3.7963809639 \\ \mathrm{H} & 4.7951087171 & -1.1359037862 & 4.5416586743 \\ \mathrm{H} & 5.8058015540 & -2.1876154406 & 5.5440199761 \\ \mathrm{O} & 2.1897992955 & -2.9769338494 & 6.0862215121 \\ \mathrm{H} & 1.5269966632 & -2.9223200752 & 5.4164540657 \\ \mathrm{H} & 1.8985427662 & -3.6287736899 & 6.7035974279 \\ \mathrm{O} & .5479410236 & -2.7965684649 & 3.8867841344 \\ \mathrm{H} & 1.1888700555 & -2.9940274060 & 3.2249512129 \\ \mathrm{C} & -.1203130681 & -1.6027935939 & 3.5837360080 \\ \mathrm{H} & -.8379300279 & -1.4305252984 & 4.3738319542 \\ \mathrm{H} & .5619414298 & -.7572520217 & 3.5457714961 \\ \mathrm{H} & -.6547808104 & -1.6684559923 & 2.6393388265 \\ \mathrm{O} & 2.7140876209 & -3.2394429871 & 2.2143667351 \\ \mathrm{H} & 2.8639897695 & -3.9978618463 & 1.6728793228 \\ \mathrm{H} & 3.3821105248 & -3.2458479832 & 2.8811372418\end{array}$

Isomer 2:

$\begin{array}{llcc}\mathrm{O} & 5.3454064268 & -1.0430465877 & 5.5753550038 \\ \mathrm{H} & 5.0559742974 & -.7738415207 & 4.7200416788 \\ \mathrm{C} & 4.6291607620 & -.3672923211 & 6.5722747177 \\ \mathrm{H} & 5.0035285474 & -.7159701802 & 7.5247315225 \\ \mathrm{H} & 4.7763152192 & .7083411494 & 6.5156069728 \\ \mathrm{H} & 3.5646923956 & -.5817285689 & 6.5195696137 \\ \mathrm{O} & 3.8321587360 & -3.2694305239 & 2.7394497938 \\ \mathrm{H} & 4.2241398262 & -3.5734849736 & 3.5424317578 \\ \mathrm{H} & 2.9726104162 & -3.6566611132 & 2.6935401752 \\ \mathrm{O} & 5.0382465601 & -3.7494222399 & 5.1931322531 \\ \mathrm{H} & 5.2138630264 & -2.8537076542 & 5.4334088575 \\ \mathrm{H} & 5.8427050340 & -4.2267835816 & 5.3190342297 \\ \mathrm{O} & 4.3730164918 & -.5695011704 & 3.0520428116 \\ \mathrm{H} & 4.1003353543 & -1.4473060913 & 2.8449041720 \\ \mathrm{C} & 5.1201530228 & -.0251233120 & 1.9989805377 \\ \mathrm{H} & 5.4066835764 & .9800718215 & 2.2887593550 \\ \mathrm{H} & 6.0237398633 & -.5991535770 & 1.8095795799 \\ \mathrm{H} & 4.5378255338 & .0318001099 & 1.0827339750\end{array}$




$\begin{array}{cccc}\text { Isomer } 3: & & & \\ \mathrm{O} & 3.3966272126 & -.8703947798 & 5.9235166710 \\ \mathrm{H} & 3.1470789367 & -1.0334752151 & 5.0296860089 \\ \mathrm{C} & 4.6039136970 & -.1609908255 & 5.9755301880 \\ \mathrm{H} & 4.8365200759 & -.0050505404 & 7.0197905245 \\ \mathrm{H} & 5.4186035702 & -.7175863815 & 5.5190931088 \\ \mathrm{H} & 4.5230042404 & .8086573101 & 5.4906703269 \\ \mathrm{O} & 3.0613344232 & -4.3318265220 & 4.1500095706 \\ \mathrm{H} & 3.1597102623 & -4.2052377449 & 5.0801575559 \\ \mathrm{H} & 2.4355568728 & -5.0291356939 & 4.0358137033 \\ \mathrm{O} & 3.4428355976 & -3.4846345764 & 6.7794495185 \\ \mathrm{H} & 3.4092169140 & -2.5570154233 & 6.6083626503 \\ \mathrm{H} & 2.9104733189 & -3.6390593684 & 7.5434013992 \\ \mathrm{O} & 2.7866009835 & -1.6903353442 & 3.3570399388 \\ \mathrm{H} & 2.8187929121 & -2.6197076391 & 3.5088096543 \\ \mathrm{C} & 3.5519700074 & -1.3485170641 & 2.2341549654 \\ \mathrm{H} & 3.4795285051 & -.2735805314 & 2.1069518924 \\ \mathrm{H} & 4.5986311681 & -1.6104451674 & 2.3673732894 \\ \mathrm{H} & 3.1787209785 & -1.8293182891 & 1.3333950721\end{array}$

\section{EFP2 structures for the three lowest energy minima $(\AA)$ for $n=3$}

\begin{tabular}{|c|c|c|c|}
\hline \multicolumn{4}{|c|}{ FRAGNAME=MEOH } \\
\hline 01 & 3.3112953328962 & -.5808287826331 & 7.0187886219120 \\
\hline $\mathrm{H} 2$ & 3.0917556414827 & -1.2732940713342 & 7.6188696517105 \\
\hline $\mathrm{C} 3$ & 4.1469249523634 & .3590406727676 & 7.6367438726531 \\
\hline $\mathrm{H} 4$ & 4.3627513727749 & 1.1235936795781 & 6.9034091669910 \\
\hline H5 & 3.6650856032997 & .8262639746342 & 8.4919551730535 \\
\hline $\mathrm{H} 6$ & 5.0850785499931 & -.0858077207245 & 7.9589718580679 \\
\hline \multicolumn{4}{|c|}{ FRAGNAME=WATER } \\
\hline 01 & .9710415917573 & -.3114465905096 & 4.9567886865316 \\
\hline $\mathrm{H} 2$ & 1.4969927577559 & -.7569149212772 & 4.3119539857475 \\
\hline $\mathrm{H} 3$ & 1.5210437219643 & -.1816082891706 & 5.7127768652360 \\
\hline \multicolumn{4}{|c|}{ FRAGNAME $=$ MEOH } \\
\hline 01 & -1.0182299818944 & -2.4869980513516 & 5.7331647999170 \\
\hline $\mathrm{H} 2$ & -.5510177464228 & -1.7023059255720 & 5.5012723911346 \\
\hline $\mathrm{C} 3$ & -2.1854722198771 & -2.6088945140833 & 4.9675363284719 \\
\hline $\mathrm{H} 4$ & -2.6749794006538 & -3.5218810684344 & 5.2769557946360 \\
\hline $\mathrm{H} 5$ & -2.8659959747820 & -1.7777704043574 & 5.1347884545823 \\
\hline $\mathrm{H} 6$ & -1.9654651844120 & -2.6773507824849 & 3.9051033733237 \\
\hline \multicolumn{4}{|c|}{ FRAGNAME=MEOH } \\
\hline 01 & 3.4508572122584 & -2.1367609158842 & 4.35 \\
\hline $\mathrm{H} 2$ & 3.67 & -1.66626 & 10932 \\
\hline $\mathrm{C} 3$ & 4.6095000842793 & -2.5170743194472 & 3.7091608094977 \\
\hline $\mathrm{H} 4$ & 4.2918193491768 & -3.0493132319789 & 2.8233262735764 \\
\hline H5 & 5.1974058230157 & -1.6554787265757 & 3.4027737713398 \\
\hline $\mathrm{H} 6$ & 5.2346494720453 & -3.1780728252068 & 4.3042426180601 \\
\hline \multicolumn{4}{|c|}{ FRAGNAME=WATER } \\
\hline 01 & 1.5810568847822 & -4.1075717980468 & 5.9223731520772 \\
\hline $\mathrm{H} 2$ & .6991896314151 & -3.7855121793380 & 5.8250787354671 \\
\hline H3 & 2.1142142622477 & -3.6281570744776 & 5.3085464764213 \\
\hline \multicolumn{4}{|c|}{ FRAGNAME=WATER } \\
\hline 01 & 2.5899745534429 & -3.0651871876202 & 8.5325588690903 \\
\hline $\mathrm{H} 2$ & 2.2333453854166 & -3.5278386615215 & 7.7911765761555 \\
\hline $\mathrm{H} 3$ & 1.9340160706434 & -3.0927561930804 & 9.2106715978080 \\
\hline \multicolumn{4}{|c|}{ Isomer 2} \\
\hline \multicolumn{4}{|c|}{ FRAGNAME=MEOH } \\
\hline 01 & 1.4733903652558 & 1.2934697364129 & 5.5653739244135 \\
\hline
\end{tabular}




\begin{tabular}{|c|c|c|c|}
\hline $\mathrm{H} 2$ & 1.1183511274771 & .4717505552052 & 5.2712178070906 \\
\hline C3 & 2.7767167416876 & 1.1208069030534 & 6.0501708391294 \\
\hline $\mathrm{H} 4$ & 3.1281253895315 & 2.0917141307252 & 6.3707223201343 \\
\hline H5 & 2.8041170997448 & .4448040485689 & 6.9011247165829 \\
\hline H6 & 3.4471181467964 & .7472376167329 & 5.2801961091098 \\
\hline \multicolumn{4}{|c|}{ FRAGNAME $=$ MEOH } \\
\hline 01 & -.1686332518711 & .1702228554108 & 1.7100823639570 \\
\hline $\mathrm{H} 2$ & .0163938982672 & 1.0518243173581 & 1.9863918109358 \\
\hline C3 & .4947185524875 & -.1185907278901 & .5100610125637 \\
\hline $\mathrm{H} 4$ & .2452403838855 & -1.1368266779754 & .2457537522696 \\
\hline H5 & .1736266227970 & .5365231712752 & -.2959035600162 \\
\hline $\mathrm{H} 6$ & 1.5739043135078 & -.0437322078849 & .6177988226226 \\
\hline \multicolumn{4}{|c|}{ FRAGNAME $=$ MEOH } \\
\hline 01 & .3199374578672 & -1.1967209570226 & 4.3506945975044 \\
\hline $\mathrm{H} 2$ & .1827558763184 & -.8886176813452 & 3.4708896874895 \\
\hline C3 & 5765 & -1.8053313164364 & 4.8453917825348 \\
\hline $\mathrm{H} 4$ & 3576 & -2.1360991104127 & 5.8513499589038 \\
\hline H5 & -1.6760995334827 & -1.1100222983284 & 4.8832515823726 \\
\hline $\mathrm{H} 6$ & -1.1277486 & -2.6697023601068 & 4.2515590734678 \\
\hline \multicolumn{4}{|c|}{ FRAGNAME=WATER } \\
\hline 01 & -.3607490223971 & 3.3576452157831 & 6.8526388803174 \\
\hline $\mathrm{H} 2$ & 8686551 & 2.633 & 6861362 \\
\hline H3 & -1.07439647 & 2.9865472854432 & 7.3464729623325 \\
\hline \multicolumn{4}{|c|}{ FRAGNAME=WATER } \\
\hline 01 & -1.0761814081294 & 4.8447079638449 & 4.2917560791434 \\
\hline $\mathrm{H} 2$ & -.9098 & 62997 & 60720142 \\
\hline H3 & -.83862885 & 5.75804 & 45638892 \\
\hline \multicolumn{4}{|c|}{ FRAGNAME=WATER } \\
\hline 01 & .7561459075459 & 2.7697364972523 & 2.9207799360988 \\
\hline $\mathrm{H} 2$ & & 3.46 & 5201 \\
\hline $\mathrm{H} 3$ & 1.0817900606472 & 2.4411577556774 & 3.7435012438353 \\
\hline \multicolumn{4}{|c|}{ Isomer 3} \\
\hline \multicolumn{4}{|c|}{ FRAGNAME=MEOH } \\
\hline $\mathrm{O} 1$ & 1.22195160 & & 7.26 \\
\hline $\mathrm{H} 2$ & 2349 & 96714 & 7.1055606092004 \\
\hline C3 & 7215 & 110948 & 7.0045939290334 \\
\hline $\mathrm{H} 4$ & 2.61 & 80770 & 926955261 \\
\hline H5 & 3.249 & .5124 & 291381707 \\
\hline $\mathrm{H} 6$ & 2.817369 & .85679 & 59524 \\
\hline \multicolumn{4}{|c|}{ FRAGNAME=MEOH } \\
\hline 01 & 1.90146393 & & 65024 \\
\hline $\mathrm{H} 2$ & & -.78 & 59818 \\
\hline C3 & 1.427 & -2.5234113044555 & 4638116 \\
\hline $\mathrm{H} 4$ & & -3.524 & \\
\hline H5 & & -2.5186529267510 & 911777171 \\
\hline $\mathrm{H} 6$ & 1.830755 & -2.25 & 1.4531620520005 \\
\hline \multicolumn{4}{|c|}{ FRAGNAME=MEOH } \\
\hline 01 & 1.4176394077547 & & 6.3711184040554 \\
\hline $\mathrm{H} 2$ & & & \\
\hline C3 & 2.50 & -2.9639082193702 & 6.8795669359050 \\
\hline $\mathrm{H} 4$ & & 597927768 & 3358383483 \\
\hline H5 & 2.57 & -3.9543 & 742186026 \\
\hline $\mathrm{H} 6$ & 3.4461224114611 & -2.4377537489750 & 6.7225543755165 \\
\hline \multicolumn{4}{|c|}{ FRAGNAME=WATER } \\
\hline 01 & -.4875834354354 & 2.3129308956672 & 5.5344896157131 \\
\hline $\mathrm{H} 2$ & & 1.7642071376471 & 6.1825655997294 \\
\hline H3 & -1.3393381293505 & 2.5391357553987 & 5.8724596689650 \\
\hline \multicolumn{4}{|c|}{ FRAGNAME=WATER } \\
\hline 01 & 1.9424930881319 & 5842650221 & 4.320 \\
\hline $\mathrm{H} 2$ & & 3.5851926393751 & 4.7706502788950 \\
\hline H3 & 1.9519756445737 & 4.6183443180965 & 4.0355869146637 \\
\hline
\end{tabular}




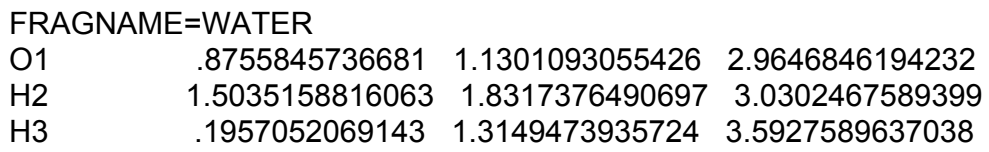

\section{EFP2 structures for the three lowest energy minima for $n=4(\AA)$}

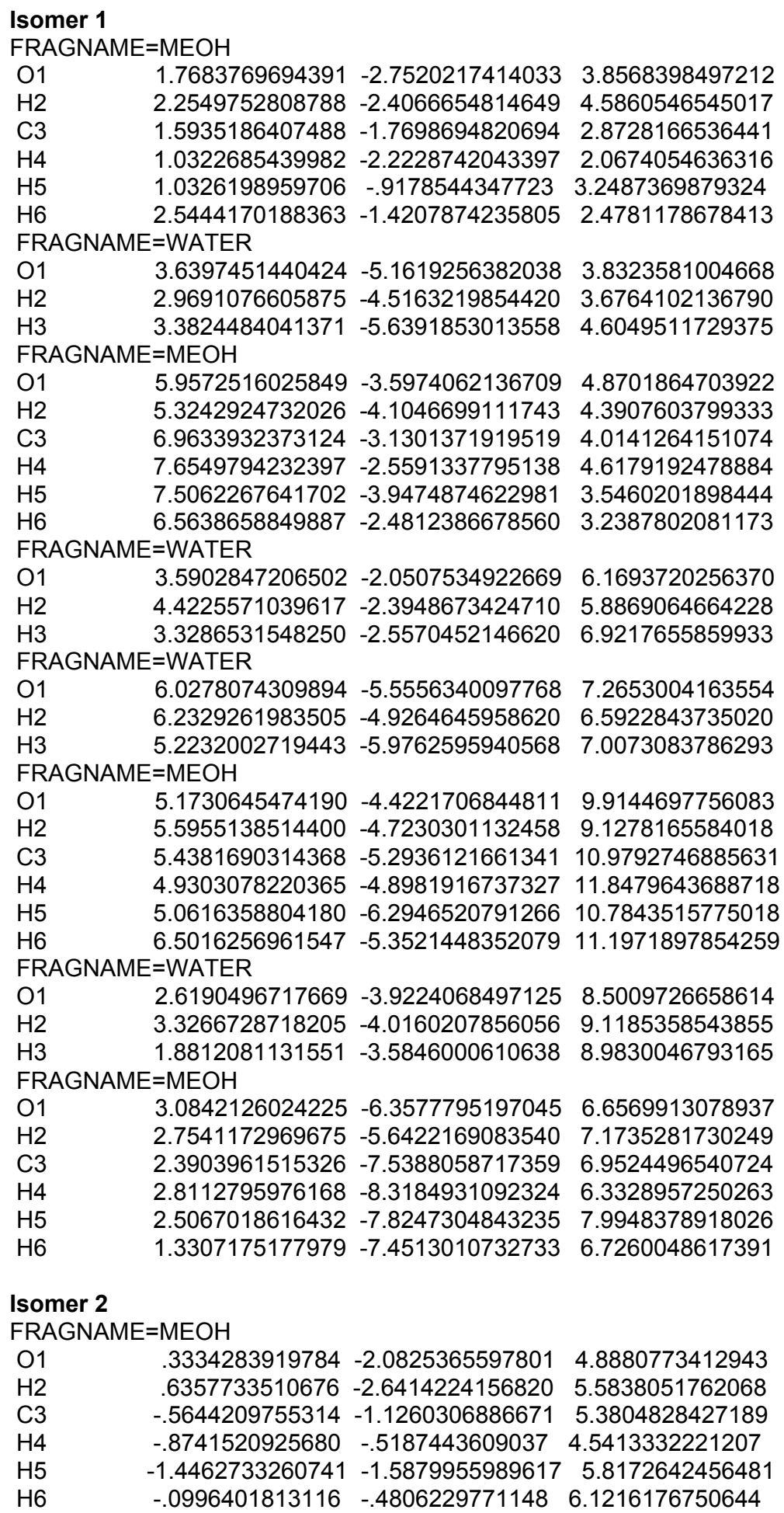




\begin{tabular}{|c|c|c|c|}
\hline \multicolumn{4}{|c|}{ FRAGNAME=WATER } \\
\hline 01 & 2.9334865079973 & -1.1777964943133 & 3.6679010762852 \\
\hline $\mathrm{H} 2$ & 2.0686141055241 & -1.4503320612307 & 3.9298143181665 \\
\hline $\mathrm{H} 3$ & 3.0731473393071 & -1.5171817804449 & 2.7983086810662 \\
\hline \multicolumn{4}{|c|}{ FRAGNAME=MEOH } \\
\hline 01 & 4.4335066002891 & -2.2428630742612 & 6.0611461470964 \\
\hline $\mathrm{H} 2$ & 4.1286579270452 & -1.8846082736500 & 5.2447370102378 \\
\hline C3 & 5.0376224610422 & -1.2470746986996 & 6.8402159025918 \\
\hline $\mathrm{H} 4$ & 5.3648006481360 & -1.7158596667292 & 7.7578744568149 \\
\hline H5 & 5.9037625402647 & -.8167561453259 & 6.3437479315880 \\
\hline $\mathrm{H} 6$ & 4.3403748334922 & -.4506922307400 & 7.0881551111189 \\
\hline \multicolumn{4}{|c|}{ FRAGNAME=WATER } \\
\hline 01 & 1.8949745608288 & -3.6270071372774 & 7.0094378112665 \\
\hline $\mathrm{H} 2$ & 2.7105947334587 & -3.1900162838878 & 6.8232089678492 \\
\hline H3 & 1.8111020672039 & -3.6559496692220 & 7.9491219011208 \\
\hline \multicolumn{4}{|c|}{ FRAGNAME=WATER } \\
\hline 01 & 5.4474770545272 & -5.0302168269941 & 6.8971860389741 \\
\hline $\mathrm{H} 2$ & 5.2727970579701 & -4.2547558190057 & 6.3882404839686 \\
\hline $\mathrm{H} 3$ & 4.758696534 & 04264 & 6.7002969995299 \\
\hline \multicolumn{4}{|c|}{ FRAGNAME $=\mathrm{MEOH}$} \\
\hline 01 & 5.1579307691933 & -4.1177059078310 & 9.7376316301101 \\
\hline $\mathrm{H} 2$ & 4043 & -4.4331037367445 & 8.8587 \\
\hline C3 & 569056054 & 0691055376 & 6344024 \\
\hline $\mathrm{H} 4$ & 368504185 & 30447205 & 20098 \\
\hline H5 & 5.7158601163525 & 17007935 & 92202 \\
\hline H6 & 7.019233605 & & 10.41 \\
\hline \multicolumn{4}{|c|}{ FRAGNAME=WATER } \\
\hline 01 & 2.1718824167578 & -3.9407 & 10.018731320647 \\
\hline $\mathrm{H} 2$ & 3.11 & & 10.0 \\
\hline $\mathrm{H} 3$ & 1.9078975723212 & 1656 & 29831 \\
\hline \multicolumn{4}{|c|}{ FRAGNAME=MEOH } \\
\hline 01 & 2.8091590333376 & -6.5 & $6.4 \varepsilon$ \\
\hline $\mathrm{H} 2$ & 202289834 & 6847 & 32696 \\
\hline C3 & 2.351 & 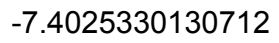 & 34716 \\
\hline $\mathrm{H} 4$ & 2.9224 & 8580 & 34050 \\
\hline H5 & 2.5072864264416 & -6.9870062215955 & 8.4816870651664 \\
\hline $\mathrm{H} 6$ & 1.2978846538585 & -7.6402985770147 & 7.3668407031011 \\
\hline \multicolumn{4}{|c|}{ Isomer 3} \\
\hline \multicolumn{4}{|c|}{ FRAGNAME=MEOH } \\
\hline 01 & 2.9366432191234 & & 4.4131392 \\
\hline $\mathrm{H} 2$ & 2.72 & -.19 & \\
\hline C3 & 317347927 & 1.2286021014738 & 57964 \\
\hline $\mathrm{H} 4$ & & & 7700 \\
\hline H5 & 48405595 & 969421 & 263741 \\
\hline $\mathrm{H} 6$ & 4.8663455627641 & .7432425639423 & 5.0080210539119 \\
\hline \multicolumn{4}{|c|}{ FRAGNAME=MEOH } \\
\hline 01 & 3.2303815851293 & & \\
\hline $\mathrm{H} 2$ & & & \\
\hline C3 & 2.25 & -1.8 & 1.389 \\
\hline $\mathrm{H} 4$ & 2.392 & & \\
\hline H5 & 67574832 & 416493517 & 1.7990793116989 \\
\hline $\mathrm{H} 6$ & 2.3738982118313 & -1.1343662483283 & .7109716858578 \\
\hline \multicolumn{4}{|c|}{$\mathrm{AME}=\mathrm{MEOH}$} \\
\hline 01 & 3.566253311 & -3.8342 & 42720 \\
\hline $\mathrm{H} 2$ & & & \\
\hline C3 & 4.9342472747762 & -3.9995269484493 & 5.0024497364812 \\
\hline $\mathrm{H} 4$ & 5.0233787759011 & -4.4557246569356 & 5.9785856700367 \\
\hline H5 & & -4.6524333023144 & 4.2713128573190 \\
\hline & 5.4596868974063 & -3.0479077979994 & 5.0161368103048 \\
\hline \multicolumn{4}{|c|}{ FRAGNAME=MEOH } \\
\hline 01 & 2.1823397766455 & -1.7812456666760 & 6.5050682611038 \\
\hline
\end{tabular}




$\begin{array}{lrrr}\text { H2 } & 2.5868711983088 & -2.4753054062333 & 6.0126878174442 \\ \text { C3 } & .7962280137556 & -1.9732240001917 & 6.5781343016681 \\ \text { H4 } & .3909462930320 & -1.1508735617277 & 7.1511863217365 \\ \text { H5 } & .3360461608430 & -1.9683929022878 & 5.5932168111193 \\ \text { H6 } & .5439659008836 & -2.9031201521851 & 7.0816321799554 \\ \text { FRAGNAME=WATER } & & \\ \text { O1 } & 2.1076677881857 & -5.4946606899591 & 6.8852730958553 \\ \text { H2 } & 2.3907201049575 & -5.2019126118964 & 6.0337694829279 \\ \text { H3 } & 2.6274389849413 & -6.2532684456970 & 7.0979480356700 \\ \text { FRAGNAME=WATER } & & \\ \text { O1 } & 3.1892608543278 & -3.5138983862574 & 8.9175299452804 \\ \text { H2 } & 3.1228494863648 & -2.6588806885038 & 8.5233057440941 \\ \text { H3 } & 2.7326654552086 & -4.1116981993522 & 8.3474119934191 \\ \text { FRAGNAME=WATER } & & \\ \text { O1 } & 5.7349868534296 & -5.0572084405960 & 8.8552642983456 \\ \text { H2 } & 6.2688514503853 & -5.0207137209395 & 9.6327829036677 \\ \text { H3 } & 5.0156279034915 & -4.4616193620747 & 8.9919200876766 \\ \text { FRAGNAME=WATER } & & \\ \text { O1 } & 4.2419782645369 & -7.4880594105252 & 7.7951248558555 \\ \text { H2 } & 4.8001741492787 & -6.8459157567726 & 8.2037167503788 \\ \text { H3 } & 4.0716786241449 & -8.1517903568762 & 8.4442301765900\end{array}$

6. EFP2 structures for the three lowest energy structures for n-5 (A)

$\begin{array}{llll}\text { Isomer 1 } & & & \\ \text { FRAGNAME=WATER } & & \\ \text { O1 } & -1.5645489110782 & -3.3918814776454 & 6.3402778105730 \\ \text { H2 } & -2.2065288550782 & -3.7634809929056 & 5.7566220851426 \\ \text { H3 } & -.9952609830771 & -4.0977089738967 & 6.6021885866693 \\ \text { FRAGNAME=MEOH } & & \\ \text { O1 } & -3.3553750760288 & -4.1740290917525 & 3.9691016536288 \\ \text { H2 } & -2.6926551558013 & -3.8835547370608 & 3.3655869056019 \\ \text { C3 } & -4.5817238310303 & -3.5575571046416 & 3.6870889477828 \\ \text { H4 } & -5.2972835495265 & -3.9282156095718 & 4.4078445904854 \\ \text { H5 } & -4.5213036914700 & -2.4764626264636 & 3.7842345932564 \\ \text { H6 } & -4.9366497496330 & -3.8035530261806 & 2.6894082676988 \\ \text { FRAGNAME=MEOH } & & \\ \text { O1 } & -1.5222248775223 & -7.7953718535405 & 1.0944433477643 \\ \text { H2 } & -.8867717106887 & -7.1006518441205 & 1.1314092206541 \\ \text { C3 } & -.8963213703041 & -9.0420435156295 & 1.2269375462818 \\ \text { H4 } & -1.6714757211363 & -9.7940766142574 & 1.1770969458319 \\ \text { H5 } & -.1859239421571 & -9.2238625194003 & .4243601454064 \\ \text { H6 } & -.3836682028540 & -9.1364552195281 & 2.1809429449001 \\ \text { FRAGNAME=WATER } & & \\ \text { O1 } & -2.8134519865526 & -7.2198177262562 & 3.8039756517295 \\ \text { H2 } & -3.1281361078852 & -6.3325464786035 & 3.8718143644049 \\ \text { H3 } & -2.6511552559798 & -7.3808235694978 & 2.8882162162034 \\ \text { FRAGNAME=WATER } & & \\ \text { O1 } & .0834778108091 & -7.2343734811928 & 4.6083634878479 \\ \text { H2 } & .2579142429051 & -6.7331025839826 & 5.3888619649990 \\ \text { H3 } & -.8550426392402 & -7.2984144392159 & 4.5311846396944 \\ \text { FRAGNAME=MEOH } & & \\ \text { O1 } & .6869498316086 & -5.8449588702864 & 2.0497511307894 \\ \text { H2 } & .5938859745072 & -6.2530396760400 & 2.8939147813707 \\ \text { C3 } & 2.0397941165452 & -5.7430634842490 & 1.6991376440789 \\ \text { H4 } & 2.0819374151153 & -5.2701655010332 & .7278016700950 \\ \text { H5 } & 2.5945384738223 & -5.1312033603370 & 2.4060598656009 \\ \text { H6 } & 2.5128496462988 & -6.7193806556113 & 1.6293065106550 \\ \text { FRAGNAME=WATER } & & \\ \text { O1 } & -1.0044802502808 & -3.3012847306542 & 2.3373905638267 \\ \text { H2 } & -.4680286641400 & -4.0594793479408 & 2.1693457123370 \\ \text { H3 } & -.5124490248613 & -2.7444291060918 & 2.9193661137195\end{array}$




\begin{tabular}{|c|c|c|c|}
\hline \multicolumn{4}{|c|}{ FRAGNAME=MEOH } \\
\hline 01 & .8079843954027 & -5.2922761753514 & 6.8963619731568 \\
\hline H2 & 1.4485710694589 & -4.7724164486018 & 6.4411722632432 \\
\hline C3 & 1.2969932119842 & -5.6907775828112 & 8.1475882323133 \\
\hline $\mathrm{H} 4$ & .5225912637423 & -6.2799312608953 & 8.6188649033447 \\
\hline H5 & 2.1899101495581 & -6.3039296104650 & 8.0548678245075 \\
\hline $\mathrm{H} 6$ & 1.5184041076668 & -4.8378396183257 & 8.7842413439329 \\
\hline \multicolumn{4}{|c|}{ FRAGNAME=WATER } \\
\hline 01 & 2.5328685987331 & -3.4548073422236 & 5.1725696391352 \\
\hline $\mathrm{H} 2$ & 1.9199231398398 & -2.7648946215921 & 4.9745879318981 \\
\hline H3 & 3.3407662207177 & -3.0361852368321 & 5.4234389663490 \\
\hline \multicolumn{4}{|c|}{ FRAGNAME=MEOH } \\
\hline 01 & .1583373453604 & -1.6425908777397 & 4.6764702741534 \\
\hline H2 & -.4279013544778 & -2.0897493016416 & 5.2631359585353 \\
\hline C3 & -.0087878930073 & -.2549886879307 & 4.7771155104164 \\
\hline $\mathrm{H} 4$ & .6860573691049 & .2001429676601 & 4.0850552729380 \\
\hline H5 & -1.0164492165283 & .0504267697365 & 4.5065731001667 \\
\hline $\mathrm{H} 6$ & .2133628157905 & .1063218678604 & 5.7780934748977 \\
\hline \multicolumn{4}{|c|}{ Isomer 2} \\
\hline \multicolumn{4}{|c|}{ FRAGNAME=MEOH } \\
\hline 01 & 1.0545736202241 & -3.9531855181063 & 7.4346394969057 \\
\hline $\mathrm{H} 2$ & .3668121842420 & -4.4349611931979 & 7.8620497220426 \\
\hline C3 & 2.1275823651104 & -4.7989806830618 & 7.1235399655975 \\
\hline $\mathrm{H} 4$ & 2.8800472937653 & -4.1929433184546 & 6.6383416779423 \\
\hline H5 & 2.5630216921434 & -5.2425937650347 & 17775107 \\
\hline $\mathrm{H} 6$ & 1.8309410 & -5.5928320916362 & 6.4426163169995 \\
\hline \multicolumn{4}{|c|}{ FRAGNAME=MEOH } \\
\hline 01 & -2.4965846397341 & -4.4590667031704 & 1.8645812986466 \\
\hline $\mathrm{H} 2$ & 56819 & -4.08 & 2.6 \\
\hline C3 & -3.2425028277684 & -3.9053952894256 & .8155082943203 \\
\hline $\mathrm{H} 4$ & -2.9024776072048 & -4.3731102049114 & -.0980166050185 \\
\hline H5 & -4.3057199719840 & -4.1014440465697 & .9294702595290 \\
\hline $\mathrm{H} 6$ & -3.0877052769453 & -2.8324987511520 & .7331440002219 \\
\hline \multicolumn{4}{|c|}{ FRAGNAME=WATER } \\
\hline 01 & -2.2968043524723 & -7.4863048363336 & 2.4427462470793 \\
\hline $\mathrm{H} 2$ & -2.416 & -6.5 & \\
\hline $\mathrm{H} 3$ & -1.3893989218245 & -7.5823009186925 & 2.6841558381691 \\
\hline \multicolumn{4}{|c|}{ FRAGNAME=WATER } \\
\hline 01 & -3.637144074 & -8.15950 & \\
\hline $\mathrm{H} 2$ & 5428255 & -7.9910659866048 & 7391189 \\
\hline H3 & -4.151899428 & -8.9502724078675 & 5.0009955348849 \\
\hline \multicolumn{4}{|c|}{ FRAGNAME=WATER } \\
\hline 01 & -.8196748677535 & -8.1019711910764 & 6.2471893825270 \\
\hline $\mathrm{H} 2$ & -1.71 & & 5.9 \\
\hline $\mathrm{H} 3$ & -.3103861203477 & -7.8603782934813 & 5.4901317244421 \\
\hline \multicolumn{4}{|c|}{ FRAGNAME=WATER } \\
\hline 01 & .6039575291409 & -7.2509341809356 & 9703377 \\
\hline $\mathrm{H} 2$ & 1.3765 & -7.6917564721167 & 371734546 \\
\hline & .7022308603719 & 38271829853 & 3.3553353066035 \\
\hline \multicolumn{4}{|c|}{ FRAGNAME=MEOH } \\
\hline 01 & -2.7198735216504 & & \\
\hline $\mathrm{H} 2$ & -1.9719260782388 & -2.9151255956954 & 4.7711989243523 \\
\hline $\mathrm{C} 3$ & 194410255 & -4.5709076177529 & 5.5448876286135 \\
\hline $\mathrm{H} 4$ & 809217657 & -5.1776707401918 & 5.3838497819398 \\
\hline H5 & -1.7540904493889 & -5.1780615039611 & 5.3463495785045 \\
\hline & & -4.2543154811469 & 6.5847702306803 \\
\hline \multicolumn{4}{|c|}{ FRAGNAME=WATER } \\
\hline 01 & .4792141875889 & & \\
\hline $\mathrm{H} 2$ & .4336299699758 & -3.7628255695768 & 3.4511833415105 \\
\hline H3 & -.3482160535557 & -4.3710350606334 & 2.3054700614966 \\
\hline & & & \\
\hline
\end{tabular}




$\begin{array}{llll}\text { O1 } & .1379293382777 & -2.4009205990336 & 5.0700740218251 \\ \text { H2 } & .3951788398722 & -2.8590250697444 & 5.8522287906081 \\ \text { C3 } & .6908627761516 & -1.1135398504384 & 5.0496885497409 \\ \text { H4 } & .3622560602052 & -.6406360585155 & 4.1346575315131 \\ \text { H5 } & .3495022914781 & -.5147583236537 & 5.8903947071650 \\ \text { H6 } & 1.7776293319051 & -1.1413920802687 & 5.0536693928245 \\ \text { FRAGNAME=MEOH } & & \\ \text { O1 } & -.7141131068004 & -6.1432377818246 & 8.5367863052078 \\ \text { H2 } & -.7110144256855 & -6.7779807364851 & 7.8404423713864 \\ \text { C3 } & -1.8685125290037 & -6.2775294206594 & 9.3196195414724 \\ \text { H4 } & -1.8150971808065 & -5.5274997627385 & 10.0964750282363 \\ \text { H5 } & -2.7716769285824 & -6.1096318596492 & 8.7382783191370 \\ \text { H6 } & -1.9264599283406 & -7.2569427663225 & 9.7878553130337\end{array}$

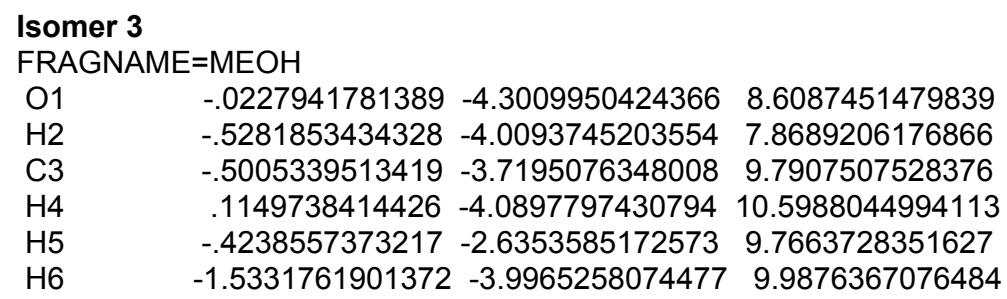




$\begin{array}{lrrr}\text { H3 } & 4.9003096606419 & -5.3488864133048 & 5.6351820284266 \\ \text { FRAGNAME=MEOH } & & \\ \text { O1 } & 2.7222861182230 & -3.3508826518585 & 7.6424303082288 \\ \text { H2 } & 1.9171960655277 & -3.7477971482094 & 7.9289472957240 \\ \text { C3 } & 3.3226342206944 & -2.6431238155106 & 8.6922682630937 \\ \text { H4 } & 4.2358676700606 & -2.2132421428082 & 8.3048298177472 \\ \text { H5 } & 2.6865259243368 & -1.8379795872910 & 9.0513812641332 \\ \text { H6 } & 3.5733584652910 & -3.2942108673261 & 9.5259805303971\end{array}$

7. EFP2 structures for the three lowest energy structures for $n=6(\AA)$

\begin{tabular}{|c|c|c|c|}
\hline \multicolumn{4}{|c|}{ FRAGNAME=WATER } \\
\hline 01 & -1.2517266542378 & -4.0271216650329 & 4.7308101599169 \\
\hline $\mathrm{H} 2$ & -.7030773229465 & -3.9214774183693 & 3.9700857298582 \\
\hline H3 & -.8917714095022 & -4.7445735845147 & 5.2273717563849 \\
\hline \multicolumn{4}{|c|}{ FRAGNAME $=M E O H$} \\
\hline 01 & .2056077448605 & -3.5717835836006 & 2.1105945321748 \\
\hline $\mathrm{H} 2$ & .4312352623884 & -4.4178990879855 & 1.7627671740511 \\
\hline C3 & -.5798068776516 & -2.8575092956826 & 1.1960256400687 \\
\hline $\mathrm{H} 4$ & -.8052445592147 & -1.9018575965467 & 1.6485848253356 \\
\hline H5 & -.0519014845575 & -2.6819579239411 & .2620284493515 \\
\hline $\mathrm{H} 6$ & -1.5153636923736 & -3.3684556942995 & .9826706197616 \\
\hline \multicolumn{4}{|c|}{ FRAGNAME=MEOH } \\
\hline 01 & -.3828030210988 & -6.3336064795638 & .9239891061259 \\
\hline $\mathrm{H} 2$ & .1955885768527 & -6.9251068690988 & 1.3749808389600 \\
\hline C3 & -.2098423834300 & -6.4381665160084 & -.4626081543072 \\
\hline $\mathrm{H} 4$ & -.8957856887373 & -5.7402505543868 & -.9222789353202 \\
\hline H5 & .801850459 & -6.17779050 & -.7634986073796 \\
\hline $\mathrm{H} 6$ & -.4397943580400 & -7.4375754974371 & -.8233808219734 \\
\hline \multicolumn{4}{|c|}{ FRAGNAME=WATER } \\
\hline 01 & -2.7932325152288 & -6.203 & 2.920107 \\
\hline $\mathrm{H} 2$ & -2.17 & -6.1 & 943 \\
\hline $\mathrm{H3}$ & -2.6579641102241 & 12892 & 3.4221224855308 \\
\hline \multicolumn{4}{|c|}{ FRAGNAME=WATER } \\
\hline 01 & -1.2999149384878 & -8.2841145400561 & 4.6056060723408 \\
\hline $\mathrm{H} 2$ & -1.0365222987316 & -7.7804469932819 & 5.3591455694584 \\
\hline H3 & -1.9422275093639 & -7.7 & 68743 \\
\hline \multicolumn{4}{|c|}{ FRAGNAME=MEOH } \\
\hline 01 & 1.0557281878676 & -8.2820665563120 & 2.8003786734773 \\
\hline $\mathrm{H} 2$ & .3157014479083 & -8.3709644139900 & 3665 \\
\hline C3 & 1.67101 & -9.5240915962080 & 28207 \\
\hline $\mathrm{H} 4$ & 2.507314 & 86024032806 & 8366 \\
\hline H5 & 2.0459492631809 & -9.9469971200294 & 3.5234040605794 \\
\hline $\mathrm{H} 6$ & .9948729571055 & -10.2368658622577 & 2.1292947853530 \\
\hline \multicolumn{4}{|c|}{ FRAGNAME=WATER } \\
\hline 01 & 2.391320357 & -6.1 & 2589 \\
\hline $\mathrm{H} 2$ & 2.1010757704460 & -6.7054600626976 & 3.9020278906808 \\
\hline H3 & 2.5483652761774 & -5.3164690049055 & 4.3076100019232 \\
\hline \multicolumn{4}{|c|}{ FRAGNAME=MEOH } \\
\hline 01 & .0355973827958 & -6.1849540322905 & 929736 \\
\hline $\mathrm{H} 2$ & .8701989 & -6.2120115185089 & 3084562 \\
\hline C3 & .1983230713918 & -5.8573498907214 & 7.7982437609270 \\
\hline $\mathrm{H} 4$ & -.7871806229309 & -5.8418493993322 & 8.2425908524469 \\
\hline H5 & .8022079766157 & -6.5943104375295 & 8.3217562384642 \\
\hline $\mathrm{H} 6$ & .6499004636124 & -4.8764789060610 & 7.9240337080969 \\
\hline \multicolumn{4}{|c|}{ FRAGNAME=WATER } \\
\hline 01 & 2.5681974754429 & -1.4497906329944 & 6.0814103124457 \\
\hline $\mathrm{H} 2$ & 1.6679026632893 & -1.5757908551466 & 6.3353229928978 \\
\hline H3 & 3.0925114422820 & -1.6200119249896 & 6.8475682791840 \\
\hline \multicolumn{4}{|c|}{ FRAGNAME=MEOH } \\
\hline 01 & -.3693184676644 & -1.8623346477785 & 6.694724266947 \\
\hline
\end{tabular}




\begin{tabular}{|c|c|c|c|}
\hline $\mathrm{H} 2$ & -.7391553573522 & -2.4734325370099 & 6.0802463265060 \\
\hline C3 & -1.1886241642524 & -.7322995923571 & 6.8182519768020 \\
\hline $\mathrm{H} 4$ & -.7149954286753 & -.0724388665141 & 7.5318077054069 \\
\hline H5 & -1.2916309772525 & -.2037858122879 & 5.8738389732922 \\
\hline H6 & -2.1776235536707 & -.9899064884260 & 7.1888893134947 \\
\hline \multicolumn{4}{|c|}{ FRAGNAME=WATER } \\
\hline 01 & 2.7728812744945 & -3.3006791410157 & 3.6554660097962 \\
\hline $\mathrm{H} 2$ & 2.8841095354800 & -2.7087988580267 & 4.3822290101165 \\
\hline H3 & 1.9539145114382 & -3.0745485359550 & 3.2443251038630 \\
\hline \multicolumn{4}{|c|}{ FRAGNAME=MEOH } \\
\hline 01 & 2.7286761765951 & -5.3391953338556 & 1.3864881078251 \\
\hline $\mathrm{H} 2$ & 3.1567737441733 & -4.6681671222357 & 1.8907268472428 \\
\hline C3 & 3.6320519522330 & -6.3583099528762 & 1.0566127566181 \\
\hline $\mathrm{H} 4$ & 3.0860355896995 & -7.0938405534003 & .4823628092629 \\
\hline H5 & 4.4546528540713 & -5.9872760701054 & .4503828047214 \\
\hline $\mathrm{H} 6$ & 4.0358868711488 & -6.8415475576513 & 1.9427571670378 \\
\hline \multicolumn{4}{|c|}{ Isomer 2} \\
\hline \multicolumn{4}{|c|}{ FRAGNAME=WATER } \\
\hline 01 & -.1538236909776 & -4.6511437365636 & 6.8294236285166 \\
\hline $\mathrm{H} 2$ & .475775928 & 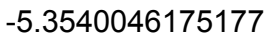 & 6.8077590128311 \\
\hline H3 & -.1183910 & -4.2291047788790 & 5.9859154195523 \\
\hline \multicolumn{4}{|c|}{ FRAGNAME=MEOH } \\
\hline 01 & -.9413257989556 & -4.6429887539019 & 1.7467211250038 \\
\hline $\mathrm{H} 2$ & 833 & -5.33692 & 09808 \\
\hline C3 & -.66 & -4.004 & .53066 \\
\hline $\mathrm{H} 4$ & -1.400193 & -3.2235338042881 & .4056333237311 \\
\hline H5 & .3248232 & -3.5513884642728 & .5299332570558 \\
\hline H6 & -.7387907 & -4.6892515182614 & -.31049 \\
\hline \multicolumn{4}{|c|}{ FRAGNAME $=\mathrm{MEOH}$} \\
\hline 01 & -3.5874904317521 & -5.3486825346412 & 3.1111669159121 \\
\hline $\mathrm{H} 2$ & -2.8 & -5.1570082579818 & 55113 \\
\hline C3 & -4.3 & -6.300 & 7466134 \\
\hline $\mathrm{H} 4$ & -5.2 & -6.4776047990798 & 3.1038221500222 \\
\hline H5 & -4.7 & -5.943 & 57691 \\
\hline H6 & -3.8639607440461 & -7.2408417733469 & 2.3246589042953 \\
\hline \multicolumn{4}{|c|}{ FRAGNAME=WATER } \\
\hline 01 & -2.4283972767175 & -6.5071154162112 & \\
\hline $\mathrm{H} 2$ & -2.8 & -6.046 & 33449 \\
\hline H3 & -1.9946225 & -5.85 & 7262200 \\
\hline \multicolumn{4}{|c|}{ FRAGNAME=WATER } \\
\hline 01 & & $-8.0 \varsigma$ & \\
\hline $\mathrm{H} 2$ & & -7.913 & 60835 \\
\hline $\mathrm{H} 3$ & -.83439411 & -7.709 & 5.0424875392201 \\
\hline \multicolumn{4}{|c|}{ FRAGNAME=MEOH } \\
\hline 01 & 1.0778108428528 & -6.8563071886471 & 2.3752145222652 \\
\hline $\mathrm{H} 2$ & & -7.26 & \\
\hline C3 & & -7.823 & 30624707 \\
\hline $\mathrm{H} 4$ & & -7.297 & .5549400251539 \\
\hline H5 & & -8.502 & 1.7800706162760 \\
\hline $\mathrm{H} 6$ & .542968595 & -8.4023177069842 & 1.1106622227979 \\
\hline \multicolumn{4}{|c|}{ FRAGNAME=WATER } \\
\hline 01 & 2.1294272991103 & -4.01467 & 2.7369909311920 \\
\hline $\mathrm{H} 2$ & & -4.9522586390861 & \\
\hline \multirow{2}{*}{\multicolumn{4}{|c|}{ FRAGNAME=MEOH }} \\
\hline & & & \\
\hline 01 & & -7.0250091128706 & \\
\hline $\mathrm{H} 2$ & 2.6704873265739 & -6.8448122684621 & 6.7835961309630 \\
\hline C3 & 1.8221528757190 & -7.5389146470015 & 8.3888278982787 \\
\hline $\mathrm{H} 4$ & & -7.7245617341560 & 8.6779733236036 \\
\hline H5 & 2.3715749433002 & -8.4754764658768 & 8.4421241601149 \\
\hline $\mathrm{H} 6$ & 2.2574442111036 & -6.8332046427732 & 9.091926741823 \\
\hline
\end{tabular}




\begin{tabular}{|c|c|c|c|}
\hline \multicolumn{4}{|c|}{ FRAGNAME=WATER } \\
\hline 01 & 1.9232100596376 & -2.2024135621198 & 6.5860116131287 \\
\hline $\mathrm{H} 2$ & 1.2992856416243 & -2.1503889506959 & 5.8796900047374 \\
\hline $\mathrm{H} 3$ & 1.5417149935366 & -2.7608820010283 & 7.2443830670597 \\
\hline \multicolumn{4}{|c|}{ FRAGNAME $=$ MEOH } \\
\hline 01 & -.1213053084770 & -2.7989136021048 & 4.3984510366513 \\
\hline $\mathrm{H} 2$ & .2808832556903 & -3.1748519716064 & 3.6337826146046 \\
\hline C3 & -1.3137353000339 & -2.1470393452882 & 4.0568799392497 \\
\hline $\mathrm{H} 4$ & -1.7258438796441 & -1.7389628146393 & 4.9693174429969 \\
\hline H5 & -1.1416246260602 & -1.3308060380913 & 3.3597470648472 \\
\hline $\mathrm{H} 6$ & -2.0390458142101 & -2.8327983269358 & 3.6261684144008 \\
\hline \multicolumn{4}{|c|}{ FRAGNAME=WATER } \\
\hline 01 & 4.1451882764802 & -3.4148323576305 & 5.0581213942795 \\
\hline $\mathrm{H} 2$ & 3.5304465562064 & -2.9839914486217 & 5.6302640185846 \\
\hline H3 & 4.8889687027357 & -2.8394994686056 & 4.9765052292563 \\
\hline \multicolumn{4}{|c|}{ FRAGNAME=MEOH } \\
\hline 01 & 4.4421618678313 & -6.3836940131879 & 5.8184933501580 \\
\hline $\mathrm{H} 2$ & 4.4405806369853 & -5.4574166201338 & 91513 \\
\hline C3 & 4.6328912066409 & -7.1048223165521 & 927609 \\
\hline $\mathrm{H} 4$ & 4.6253182452800 & -8.1540187464933 & 4.8931111414720 \\
\hline H5 & $3.83609949 s$ & 440844698 & 3.9159784627298 \\
\hline $\mathrm{H} 6$ & 5.5872993214305 & -6.8709158389847 & 4.1672597581523 \\
\hline \multirow{2}{*}{\multicolumn{4}{|c|}{$\begin{array}{l}\text { Isomer } 3 \\
\text { FRAGNAME=WATER }\end{array}$}} \\
\hline & & & \\
\hline 01 & -1.0493770578674 & -4.0227361383593 & 6.2190670460604 \\
\hline $\mathrm{H} 2$ & -1.1536041525837 & -3.9395345 & 5.28 \\
\hline H3 & -.36924900 & 142 & 6.3603314031169 \\
\hline \multicolumn{4}{|c|}{ FRAGNAME=MEOH } \\
\hline 01 & -.88585334513 & -3.6956955 & 55843 \\
\hline $\mathrm{H} 2$ & -.08009342 & -4.1 & 2873 \\
\hline C3 & -.6730 & 35723 & 30548 \\
\hline $\mathrm{H} 4$ & -1.627 & -1.8825528979882 & 3684972 \\
\hline H5 & .0267 & -1.8480508600392 & 3.3551542870269 \\
\hline $\mathrm{H} 6$ & -.3073031 & -2.3 & 1.7002546744745 \\
\hline \multicolumn{4}{|c|}{ FRAGNAME $=\mathrm{MEOH}$} \\
\hline 01 & -3.4253907251745 & -5.3955499821933 & \\
\hline $\mathrm{H} 2$ & -2.643 & -4.8 & \\
\hline C3 & -3.35193978 & -6.4190976709002 & 1567434108 \\
\hline $\mathrm{H} 4$ & -4.27188201 & $-6.9 \varepsilon$ & 26992238 \\
\hline H5 & -3.26 & -6.02415 & .8223846235071 \\
\hline $\mathrm{H} 6$ & -2.51941573 & -7.0910899071094 & 2.0239864094820 \\
\hline \multicolumn{4}{|c|}{ FRAGNAME=WATER } \\
\hline 01 & -3.2604334938359 & & \\
\hline $\mathrm{H} 2$ & & & \\
\hline H3 & -3.4700748929517 & -6.0287294163344 & 4.8345162300099 \\
\hline \multicolumn{4}{|c|}{ FRAGNAME=WATER } \\
\hline 01 & -.784886770 & & \\
\hline $\mathrm{H} 2$ & 533031222 & -7.5217656556367 & 5.7601922426199 \\
\hline H3 & -1.6313397786800 & -7.6860346222765 & 5.4619794080816 \\
\hline \multicolumn{4}{|c|}{ FRAGNAME=MEOH } \\
\hline 01 & .3361045373447 & & \\
\hline $\mathrm{H} 2$ & -.1092 & & 48706 \\
\hline C3 & .9000048872202 & -9.1942075023627 & 2.1574173263763 \\
\hline $\mathrm{H} 4$ & 1.3936893881204 & -9.0030868262057 & 1.2147346463533 \\
\hline H5 & 1.6373942773685 & 6299259322 & 2.8651582788862 \\
\hline $\mathrm{H} 6$ & .1431435307204 & & 2.0026415661500 \\
\hline \multicolumn{4}{|c|}{ FRAGNAME=WATER } \\
\hline 01 & 1.5839336374677 & -5.4093280925052 & 3.4560330661685 \\
\hline $\mathrm{H} 2$ & 1.3609890525782 & -6.2307299254145 & 3.0480217979058 \\
\hline & 2.3383615145316 & -5.0748329154591 & 2.997961009708 \\
\hline \multicolumn{4}{|c|}{ FRAGNAME=MEOH } \\
\hline 01 & 1.2381129900553 & -6.0563970093935 & 6.505640942708 \\
\hline
\end{tabular}




$\begin{array}{lrll}\text { H2 } & 1.8571457030813 & -5.8287716787944 & 5.8327454801296 \\ \text { C3 } & 1.8951373587621 & -6.2815778471789 & 7.7226506859990 \\ \text { H4 } & 1.1376763644521 & -6.5370391643781 & 8.4505892081372 \\ \text { H5 } & 2.6004489198188 & -7.1059665007208 & 7.6535703689969 \\ \text { H6 } & 2.4207877210707 & -5.3945521262857 & 8.0672231857740 \\ \text { FRAGNAME=WATER } & & \\ \text { O1 } & 3.2187957582864 & -2.2404812677332 & 5.4569321524456 \\ \text { H2 } & 2.3521337578950 & -1.9506860147441 & 5.6931399899325 \\ \text { H3 } & 3.3704844922182 & -3.0484255349862 & 5.9207155534839 \\ \text { FRAGNAME=MEOH } & & \\ \text { O1 } & .4235761135455 & -1.3675741427321 & 6.3061172110165 \\ \text { H2 } & -.1192943161707 & -2.1251671584262 & 6.4444921133543 \\ \text { C3 } & .2614265298307 & -.4486892677530 & 7.3515158168509 \\ \text { H4 } & .9052341840650 & .3931765083660 & 7.1377967165155 \\ \text { H5 } & -.7632666269122 & -.0919846773830 & 7.4194783813930 \\ \text { H6 } & .5525462486323 & -.8723048691299 & 8.3094575019850 \\ \text { FRAGNAME=WATER } & & \\ \text { O1 } & 4.0093230642614 & -3.6660618265717 & 3.0055197932064 \\ \text { H2 } & 3.7262422706314 & -2.9823151137254 & 3.5913816805290 \\ \text { H3 } & 4.5336026738976 & -3.2521199222479 & 2.3386903961099 \\ \text { FRAGNAME=MEOH } & & \\ \text { O1 } & 4.1989464647822 & -5.1461225518515 & 5.7152300123124 \\ \text { H2 } & 4.3478060066682 & -4.9714743179765 & 4.8013690132105 \\ \text { C3 } & 5.3796626670660 & -5.5816153226938 & 6.3314730181578 \\ \text { H4 } & 5.1469775478401 & -5.7646387808594 & 7.3713105937201 \\ \text { H5 } & 5.7470891085920 & -6.5053433005744 & 5.8914985036753 \\ \text { H6 } & 6.1627524447924 & -4.8293366424436 & 6.2795083399250\end{array}$

\section{EFP2 structures for the three lowest energy minima $n=7$ (A)}

$\begin{array}{llll}\text { Isomer } 1 & & & \\ \text { FRAGNAME=MEOH } & & \\ \text { O1 } & 7.6296266126190 & -.4424567163955 & 3.1498399759845 \\ \text { H2 } & 7.0694836089176 & .2876082620073 & 3.3524431279832 \\ \text { C3 } & 8.4390970818528 & -.7610615640759 & 4.2483610984568 \\ \text { H4 } & 9.0551214747343 & -1.6003499818840 & 3.9567945818482 \\ \text { H5 } & 9.0889598612545 & .0658375999762 & 4.5236233023355 \\ \text { H6 } & 7.8484408129529 & -1.0498310951624 & 5.1141500279585 \\ \text { FRAGNAME=WATER } & & \\ \text { O1 } & 5.7353409447406 & -2.6129649478344 & 2.0117132810493 \\ \text { H2 } & 6.3090626947093 & -1.9938397988493 & 2.4340944567360 \\ \text { H3 } & 6.0602538885708 & -2.7281827229869 & 1.1330581170127 \\ \text { FRAGNAME=MEOH } & & \\ \text { O1 } & 3.0659639577856 & -1.3126945274582 & 1.4610087319934 \\ \text { H2 } & 3.7854462515334 & -1.8340591744287 & 1.7745804596066 \\ \text { C3 } & 3.3203597510036 & -.8542539884411 & .1615414570598 \\ \text { H4 } & 2.4651507619691 & -.2662996701511 & -.1414922690795 \\ \text { H5 } & 4.2050179343220 & -.2236160098500 & .1224418523009 \\ \text { H6 } & 3.4409264246192 & -1.6762554709290 & -.5396221333551 \\ \text { FRAGNAME=WATER } & & \\ \text { O1 } & 3.6560579983527 & 3.2205915822666 & 1.0375541446668 \\ \text { H2 } & 4.3180990164953 & 3.7276056334588 & 1.4797270704073 \\ \text { H3 } & 3.3518308232771 & 2.5745450452759 & 1.6547641187076 \\ \text { FRAGNAME=MEOH } & & \\ \text { O1 } & 2.8847939642133 & 1.1145450346610 & 3.1491275370917 \\ \text { H2 } & 2.8674585152427 & .2828277285116 & 2.7066923448359 \\ \text { C3 } & 1.7727811443846 & 1.2469070956719 & 3.9913911059264 \\ \text { H4 } & 1.8480338628923 & 2.2144511048606 & 4.4679388507952 \\ \text { H5 } & .8391672030679 & 1.2066546814485 & 3.4358752266085 \\ \text { H6 } & 1.7581854171005 & .4814270830370 & 4.7631944847244 \\ \text { FRAGNAME=WATER } & & \\ \text { O1 } & 5.7743499127273 & 1.7442597983005 & 4.0329469923426\end{array}$




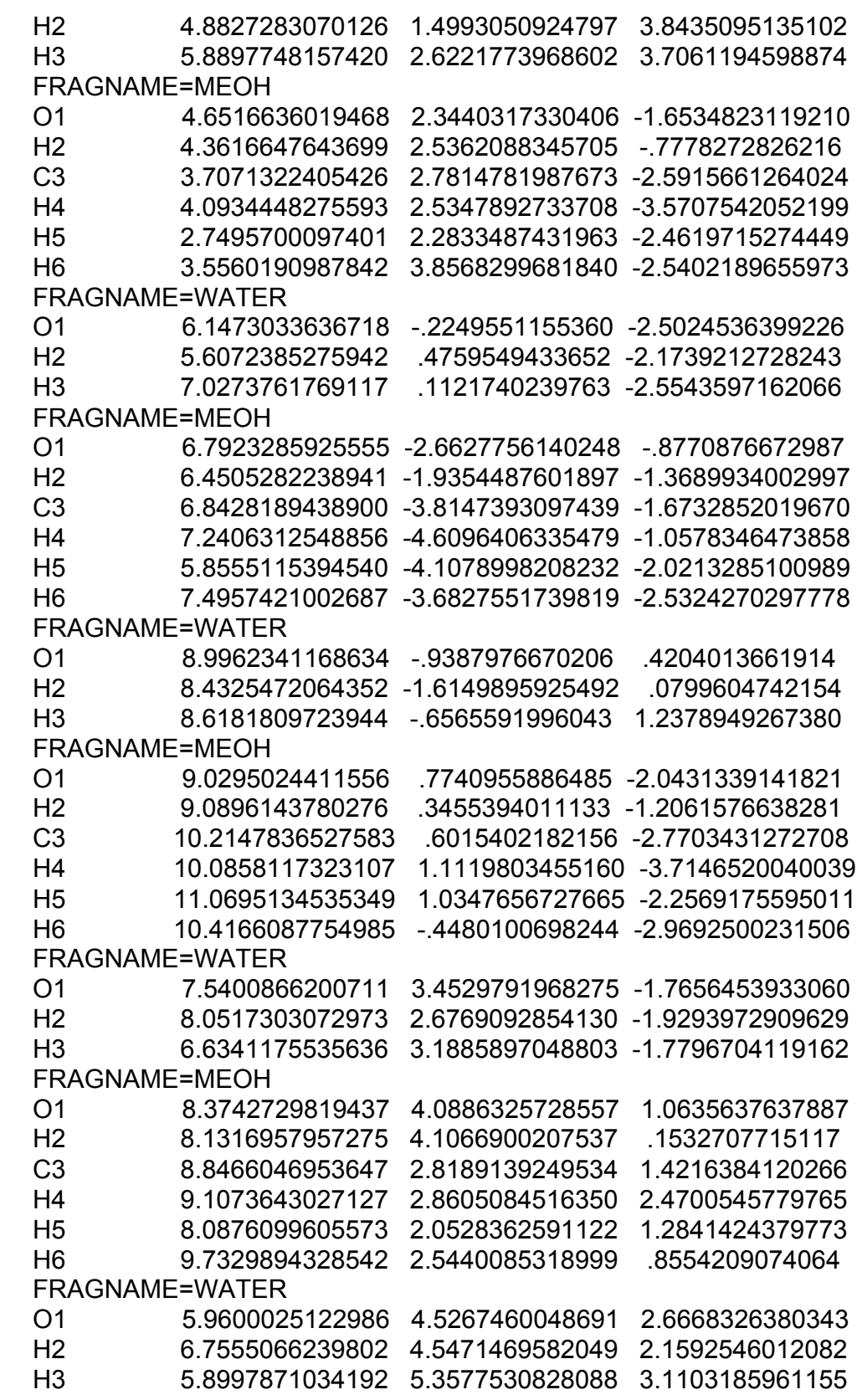

\begin{tabular}{lrcc}
\multicolumn{4}{l}{ Isomer 2 } \\
FRAGNAME=MEOH & & \\
O1 & 7.2180997338739 & .7053740851505 & 2.2878695612713 \\
H2 & 7.4347244680384 & 1.4813959606356 & 1.7993341298003 \\
C3 & 7.2958187394438 & .9532996394382 & 3.6648201129430 \\
H4 & 7.0382732800882 & .0321927578557 & 4.1689377416428 \\
H5 & 8.2991631800276 & 1.2436712191272 & 3.9662125808390 \\
H6 & 6.5960711060588 & 1.7250379657331 & 3.9756678985588 \\
FRAGNAME=WATER & & \\
O1 & 4.4181900302949 & -.0609942808020 & 1.5244479436828 \\
H2 & 5.3014144399151 & .2118436964018 & 1.7151113500141 \\
H3 & 4.4813028689251 & -.7731634135321 & .9082415253017 \\
FRAGNAME=MEOH & & \\
O1 & 2.8668950414962 & 2.3782611975836 & .5231417755405 \\
H2 & 3.3468770173216 & 1.6146970862868 & .7958875276761
\end{tabular}




\begin{tabular}{|c|c|c|c|}
\hline C3 & 2.0700895211450 & 2.0860705740043 & -.5918585600087 \\
\hline $\mathrm{H} 4$ & 1.5473217047105 & 2.9947746118766 & -.8561934161176 \\
\hline $\mathrm{H} 5$ & 2.6680657588216 & 1.7709021890266 & -1.4432964313362 \\
\hline H6 & 1.3351731991049 & 1.3158696607989 & -.3715367648562 \\
\hline \multicolumn{4}{|c|}{ FRAGNAME=WATER } \\
\hline 01 & 3.4064407435386 & 3.3207495418180 & 3.4123334046976 \\
\hline $\mathrm{H} 2$ & 3.3609245667327 & 2.4937370793140 & 3.8649448479684 \\
\hline H3 & 2.8987283698290 & 3.2259418999946 & 2.6223219423114 \\
\hline \multicolumn{4}{|c|}{ FRAGNAME=MEOH } \\
\hline 01 & 3.3834839797590 & .3805587997440 & 4.3600438973841 \\
\hline $\mathrm{H} 2$ & 3.5843678599683 & .0308507261602 & 3.5084847247906 \\
\hline C3 & 2.2796509212435 & -.2803127228308 & 4.9152929906951 \\
\hline $\mathrm{H} 4$ & 2.0989269033458 & .1619126954307 & 5.8851783670657 \\
\hline H5 & 1.3873598709165 & -.1560545375086 & 4.3068279339067 \\
\hline $\mathrm{H} 6$ & 2.4709843723673 & -1.3420255093731 & 5.0494966042683 \\
\hline \multicolumn{4}{|c|}{ FRAGNAME=WATER } \\
\hline 01 & 5.9694672841033 & 3.5910400379932 & 1.8455250200749 \\
\hline $\mathrm{H} 2$ & 5.2304154122899 & 3.6103468621296 & 2.4323026878320 \\
\hline H3 & 6.2287417060624 & 4.4878374211166 & 1.7062063859721 \\
\hline \multicolumn{4}{|c|}{ FRAGNAME=MEOH } \\
\hline 01 & 5.5701437710198 & 3.0204555258021 & -1.1171518622881 \\
\hline $\mathrm{H} 2$ & 5.2801890200028 & 3.0652228181006 & -.2217607598147 \\
\hline C3 & 5.1890017022810 & 4.1737390673023 & -1.8158420242246 \\
\hline $\mathrm{H} 4$ & 5.5474228760900 & 4.0643195357479 & -2.8299740485583 \\
\hline $\mathrm{H} 5$ & 4.1085114902503 & 4.2909086778413 & -1.8416359095071 \\
\hline $\mathrm{H} 6$ & 5.6315890563997 & 5.0694840552959 & -1.3873440767604 \\
\hline \multicolumn{4}{|c|}{ FRAGNAME=WATER } \\
\hline 01 & 5.9719781763214 & .2785560153230 & -2.4630098910788 \\
\hline $\mathrm{H} 2$ & 5.7468474626672 & 1.1052940313040 & -2.0671578116744 \\
\hline H3 & 6.9140408082718 & .2209988522720 & -2.4538652461005 \\
\hline \multicolumn{4}{|c|}{ FRAGNAME=MEOH } \\
\hline 01 & 5.1371796809793 & -1.9948038148864 & -.7236315184376 \\
\hline $\mathrm{H} 2$ & 5.3201734898490 & -1.3194723186005 & -1.3546970701242 \\
\hline C3 & 4.4727886618072 & -3.0686430155239 & -1.3310474310233 \\
\hline $\mathrm{H} 4$ & 4.2913020591399 & -3.8065093005209 & -.5619452967029 \\
\hline H5 & 3.5173534357232 & -2.7655629858772 & -1.7519264692674 \\
\hline $\mathrm{H} 6$ & 5.0747196804421 & -3.5249093025980 & -2.1129382575504 \\
\hline \multicolumn{4}{|c|}{ FRAGNAME=WATER } \\
\hline 01 & 7.9922514077924 & -1.6938841742382 & .4909725335929 \\
\hline $\mathrm{H} 2$ & 7.1328231293827 & -1.9820581092131 & .2278807786132 \\
\hline H3 & 7.8660166537392 & -1.0353080937926 & 1.1552170572449 \\
\hline \multicolumn{4}{|c|}{ FRAGNAME=MEOH } \\
\hline 01 & 8.9835003832504 & -.0820679406138 & -1.8089208462767 \\
\hline $\mathrm{H} 2$ & 8.8055865143472 & -.6978855991162 & -1.1183280855166 \\
\hline C3 & 10.1119919808929 & -.4757656283351 & -2.5403639341365 \\
\hline $\mathrm{H} 4$ & 10.2645956498924 & .2649177412623 & -3.3130232385579 \\
\hline H5 & 11.0024529158648 & $3-.5147832158838$ & -1.9179411745939 \\
\hline $\mathrm{H} 6$ & 9.9683980249778 & -1.4441527188250 & -3.0130798734910 \\
\hline \multicolumn{4}{|c|}{ FRAGNAME=WATER } \\
\hline 01 & 8.3683344505397 & 2.3743322418897 & -.0712719245885 \\
\hline $\mathrm{H} 2$ & 8.7159366901026 & 1.7205842669100 & -.6566476349903 \\
\hline H3 & 7.5685083591712 & 2.6895719850822 & -.4608621990382 \\
\hline \multicolumn{4}{|c|}{ FRAGNAME=MEOH } \\
\hline 01 & 9.7515536563498 & 4.7403309560053 & 1.2385904451894 \\
\hline $\mathrm{H} 2$ & 9.4648431550481 & 4.0160729927902 & .7084484022909 \\
\hline $\mathrm{C} 3$ & 9.8901219468567 & 4.3463198599106 & 2.5761457640697 \\
\hline $\mathrm{H} 4$ & 10.2190621501429 & 5.2140210479555 & 3.1309386312072 \\
\hline H5 & 8.9471494899433 & 4.0046665063518 & 2.9955847607671 \\
\hline $\mathrm{H} 6$ & 10.6329950665190 & 3.5608352755870 & 2.6901924053872 \\
\hline \multicolumn{4}{|c|}{ FRAGNAME=WATER } \\
\hline 01 & 7.1781628305535 & 6.2369441465478 & .9180860552041 \\
\hline $\mathrm{H} 2$ & 8.0831742820758 & 5.9776205513388 & .9857912961307 \\
\hline
\end{tabular}




\begin{tabular}{|c|c|c|c|}
\hline \\
\hline 01 & 7.3542159886897 & .3012865809043 & 1.9077399387438 \\
\hline $\mathrm{H} 2$ & 7.5581355842490 & 1.0846411415137 & 1.4254790908564 \\
\hline C3 & 7.5372129394937 & .5077872822987 & 3.2815556914431 \\
\hline $\mathrm{H} 4$ & 7.2893451017737 & -.4198604387604 & 3.7784796042679 \\
\hline H5 & 8.5675957920898 & .7618798034838 & 3.5173549878793 \\
\hline H6 & 6.8843913256132 & 1.2892719091501 & 3.6622903220822 \\
\hline \multicolumn{4}{|c|}{ FRAGNAME=WATER } \\
\hline 01 & 4.4918201522434 & -.2955653421172 & .9083221431045 \\
\hline $\mathrm{H} 2$ & 5.3161989696905 & -.0167617857852 & 1.2737624205626 \\
\hline H3 & 4.6923957250030 & -.9317269285193 & .2405300125234 \\
\hline \multicolumn{4}{|c|}{ FRAGNAME=MEOH } \\
\hline 01 & 2.8268151432446 & 1.8546631003116 & -3898078679473 \\
\hline $\mathrm{H} 2$ & 3.3543535336321 & 1.2718475535934 & .1296460565317 \\
\hline C3 & 2.4307181304424 & 1.2304071298754 & -1.5801489055783 \\
\hline $\mathrm{H} 4$ & 1.8324525979171 & 1.9438323209206 & -2.1296987066643 \\
\hline H5 & 3.2845318946170 & .9507688907298 & -2.1922417512801 \\
\hline H6 & 1.8271590029046 & .3463635108090 & -1.3903163486328 \\
\hline \multicolumn{4}{|c|}{ FRAGNAME=WATER } \\
\hline 01 & .9998389617583 & 2.9626080132221 & 1.7239760408605 \\
\hline $\mathrm{H} 2$ & .7277630735312 & 3.8526982099325 & 1.5671557397733 \\
\hline H3 & 1.4738722086078 & 2.6846331569831 & .9565772434626 \\
\hline \multicolumn{4}{|c|}{ FRAGNAME=MEOH } \\
\hline 01 & 3.3590769543503 & 2.3986550757332 & 3.5821207440630 \\
\hline $\mathrm{H} 2$ & 2.5622217956542 & 2.6214314199146 & 3.1313484611769 \\
\hline C3 & 3.2701517768343 & 1.1128852262969 & 4.1320192375406 \\
\hline $\mathrm{H} 4$ & 4.2078010573769 & .9155117245466 & 4.6327783233334 \\
\hline H5 & 2.4668691924639 & 1.0408026970389 & 4.8609872334561 \\
\hline H6 & 3.1212201600905 & .3561175071826 & 3.3658762134498 \\
\hline \multicolumn{4}{|c|}{ FRAGNAME=WATER } \\
\hline 01 & 5.6096833457504 & 2.9731285247302 & 1.6216641564237 \\
\hline $\mathrm{H} 2$ & 4.9056 & 2.8292953940175 & 4512452 \\
\hline H3 & 5.7840500235853 & 3.9007462650007 & 1.6218731152573 \\
\hline \multicolumn{4}{|c|}{ FRAGNAME=MEOH } \\
\hline 01 & 5.7053915672459 & 2.8409184217844 & -1.4041233349739 \\
\hline $\mathrm{H} 2$ & 5.3140505357438 & 2.7244458405246 & -.5549534877918 \\
\hline C3 & 84838 & 3.9947643992189 & -2.0221489281393 \\
\hline $\mathrm{H} 4$ & 5.6882520851944 & 4.0741568234220 & -2.9861339022543 \\
\hline H5 & 4.1308637438145 & 3.9354921819014 & -2.1774716958648 \\
\hline H6 & 5.4304867921087 & 4.8897791343433 & -1.4476482206819 \\
\hline \multicolumn{4}{|c|}{ FRAGNAME=WATER } \\
\hline 01 & 6.3686609853405 & .3140907796935 & -3.0007613037712 \\
\hline $\mathrm{H} 2$ & 6.0979239793871 & 1.0922944312461 & -2.5403495267877 \\
\hline H3 & 7.3074353487535 & .2624608084819 & -2.9176018500769 \\
\hline \multicolumn{4}{|c|}{ FRAGNAME=MEOH } \\
\hline 01 & 5.4019540398699 & -2.050 & \\
\hline $\mathrm{H} 2$ & & -1.348733077431 & -2.0402156526457 \\
\hline C3 & 4.6558944880838 & -3.0257779340292 & -2.1302061966134 \\
\hline $\mathrm{H} 4$ & 4.4204261569021 & -3.7990528414852 & -1.4122210177910 \\
\hline H5 & 3.7253907549924 & -2.6225098247956 & -2.5218575405713 \\
\hline H6 & 5.2192352627135 & -3.4714277756677 & -2.9462310312904 \\
\hline \multicolumn{4}{|c|}{ FRAGNAME=WATER } \\
\hline 01 & 8.1830653724717 & -1.9025795772997 & -.0784853282815 \\
\hline $\mathrm{H} 2$ & 7.3493977603110 & -2.1755584052718 & -.4268519828007 \\
\hline H3 & 7.9987837920197 & -1.3376609675643 & 6548534185118 \\
\hline \multicolumn{4}{|c|}{ FRAGNAME=MEOH } \\
\hline 01 & 9.3160065762734 & -.0073336739378 & -2.0754246906516 \\
\hline $\mathrm{H} 2$ & & & \\
\hline & & & \\
\hline
\end{tabular}




$\begin{array}{lrrr}\text { H4 } & 10.7293964907337 & .5063235424614 & -3.4029838666767 \\ \text { H5 } & 11.3439656253579 & -.4081178095640 & -2.0301318407140 \\ \text { H6 } & 10.4383941039461 & -1.2273191738634 & -3.3112154853385 \\ \text { FRAGNAME=WATER } & & & \\ \text { O1 } & 8.4397105965896 & 2.2776330768389 & -.2197243066270 \\ \text { H2 } & 8.8798731821034 & 1.6955232020273 & -.8182921357226 \\ \text { H3 } & 7.6528853229217 & 2.5678609593404 & -.6527973016334 \\ \text { FRAGNAME=MEOH } & & \\ \text { O1 } & 9.3939140793431 & 4.7141443666797 & 1.3339821716420 \\ \text { H2 } & 9.2568342516728 & 3.9915879828857 & .7449792110636 \\ \text { C3 } & 9.6040591014407 & 4.2512591032422 & 2.6397679307253 \\ \text { H4 } & 9.7542189837933 & 5.1203708523625 & 3.2650669885945 \\ \text { H5 } & 8.7458369143871 & 3.6993809013140 & 3.0149189247681 \\ \text { H6 } & 10.4868377208285 & 3.6203281830119 & 2.7067098725227 \\ \text { FRAGNAME=WATER } & & \\ \text { O1 } & 6.6057496510918 & 5.8180107703661 & 1.1186649474542 \\ \text { H2 } & 7.5347603576167 & 5.6588861343069 & 1.1686340521970 \\ \text { H3 } & 6.4408209418765 & 6.6145714340519 & 1.5973799816995\end{array}$

9. EFP2 structures for the three lowest energy structures for $n=8(\AA)$

$\begin{array}{llll}\text { Isomer 1 } & & & \\ \text { FRAGNAME=WATER } & & \\ \text { O1 } & -.9979303660238 & -1.9884236872870 & 7.4789811694884 \\ \text { H2 } & -1.5840752477997 & -2.6637054528185 & 7.7811519304436 \\ \text { H3 } & -.1371991927666 & -2.2161711786769 & 7.7922723784561 \\ \text { FRAGNAME=MEOH } & & \\ \text { O1 } & -2.8632110775477 & -4.4186966110863 & 7.8527201408913 \\ \text { H2 } & -2.9010857221725 & -4.6161142762137 & 6.9321796087712 \\ \text { C3 } & -4.1321123568964 & -4.5406457739876 & 8.4345432162153 \\ \text { H4 } & -4.0250520769219 & -4.3034094855205 & 9.4839045335820 \\ \text { H5 } & -4.8456437691066 & -3.8484751885784 & 7.9945137343288 \\ \text { H6 } & -4.5205028620299 & -5.5522075109182 & 8.3465158998712 \\ \text { FRAGNAME=MEOH } & & \\ \text { O1 } & -5.0291602472098 & -6.7441516380955 & 4.2904767085811 \\ \text { H2 } & -4.5925097227729 & -5.9187040890196 & 4.4160782918487 \\ \text { C3 } & -6.4037525296649 & -6.6148931839399 & 4.5298267766587 \\ \text { H4 } & -6.8472205018161 & -7.5880221549498 & 4.3708981827668 \\ \text { H5 } & -6.6076710797597 & -6.3045758059812 & 5.5515769860103 \\ \text { H6 } & -6.8672667500456 & -5.9077960827085 & 3.8464371385623 \\ \text { FRAGNAME=WATER } & & \\ \text { O1 } & .8883822281513 & -7.6498688340987 & 7.0050534950161 \\ \text { H2 } & -4814762714237 & -8.2491601337339 & 6.3999452942548 \\ \text { H3 } & 1.6520899042201 & -8.0864993475473 & 7.3470810294437 \\ \text { FRAGNAME=WATER } & & \\ \text { O1 } & -1.1660817379443 & -6.6700450021372 & 9.0506229644739 \\ \text { H2 } & -.4910018299022 & -6.9600770732663 & 8.4581476742363 \\ \text { H3 } & -1.5106057078973 & -5.8658019706264 & 8.6965398747030 \\ \text { FRAGNAME=MEOH } & & \\ \text { O1 } & -1.1475266193797 & -6.5937104401360 & 3.2256971282881 \\ \text { H2 } & -.8830243430256 & -7.3529594745470 & 3.7170041036460 \\ \text { C3 } & -1.8846478804190 & -6.9714651373961 & 2.0954521188913 \\ \text { H4 } & -2.1580010284795 & -6.0635866667893 & 1.5758976236232 \\ \text { H5 } & -1.2997949188259 & -7.5927561368985 & 1.4218070731149 \\ \text { H6 } & -2.7939728195681 & -7.5022800425628 & 2.3660382087347 \\ \text { FRAGNAME=WATER } & & \\ \text { O1 } & -2.8649643101029 & -4.6758436214721 & 4.8200209290157 \\ \text { H2 } & -2.2564471013302 & -5.2836066869356 & 4.4311645069864 \\ \text { H3 } & -2.5512991542033 & -3.8094879336369 & 4.6152760970968 \\ \text { FRAGNAME=MEOH } & & \\ \text { O1 } & 1.7502618418923 & -3.0235706989137 & 8.2653980511369 \\ \text { H2 } & 1.9047139266698 & -3.6157318953758 & 7.5489535205566\end{array}$




\begin{tabular}{|c|c|c|c|}
\hline C3 & 2.0028595487865 & -3.6564262358893 & 9.4898115490352 \\
\hline $\mathrm{H} 4$ & 1.8094874036023 & -2.9316307283732 & 10.2683876983300 \\
\hline H5 & 1.3499434255908 & -4.5119102247264 & 9.6437622598503 \\
\hline $\mathrm{H} 6$ & 3.0369902524319 & -3.9821055655989 & 9.5695599189261 \\
\hline \multicolumn{4}{|c|}{ FRAGNAME=WATER } \\
\hline 01 & .8132715810896 & -4.1753589648252 & 3.5061599838230 \\
\hline $\mathrm{H} 2$ & .2232846982757 & -3.4832522451243 & 3.7587114057550 \\
\hline $\mathrm{H} 3$ & .2774787191129 & -4.9138773776130 & 3.2645093742733 \\
\hline \multicolumn{4}{|c|}{ FRAGNAME $=$ MEOH } \\
\hline 01 & -1.2616889031889 & -2.1083655374960 & 4.5262318798363 \\
\hline $\mathrm{H} 2$ & -1.1070700242870 & -2.0026447284868 & 5.4496598452539 \\
\hline C3 & -1.4683919150089 & -.8637933535060 & 3.9164662775039 \\
\hline $\mathrm{H} 4$ & -1.6392631427989 & -1.0491109955128 & 2.8651040518301 \\
\hline H5 & -2.3383481249579 & -.3547629973531 & 4.3237959102732 \\
\hline $\mathrm{H} 6$ & -.6007930511432 & -.2164814588566 & 4.0170280146938 \\
\hline \multicolumn{4}{|c|}{ FRAGNAME=WATER } \\
\hline 01 & 2.1626888459661 & -5.0780844707045 & 6.0502445020756 \\
\hline $\mathrm{H} 2$ & 1.8611285941313 & -4.7644849103872 & 5.2126317130438 \\
\hline $\mathrm{H} 3$ & 1.6674100862198 & -5.8583124932286 & 6.2421341502587 \\
\hline \multicolumn{4}{|c|}{ FRAGNAME=MEOH } \\
\hline 01 & 4.8105153689204 & -5.8764195333404 & 7.2773918830590 \\
\hline $\mathrm{H} 2$ & 4.1118359811327 & -5.5130311042707 & 6.7600921080884 \\
\hline C3 & 6.0341374637337 & -5.7559475809709 & 6.6052790771837 \\
\hline $\mathrm{H} 4$ & 6.7919466686762 & -6.1874913117425 & 7.2443388176113 \\
\hline H5 & 6.0302280849535 & -6.2946477950809 & 5.6610121993778 \\
\hline H6 & 6.2906569916967 & -4.7162363081258 & 6.4180590424247 \\
\hline \multicolumn{4}{|c|}{ FRAGNAME=MEOH } \\
\hline 01 & -3.6318989562058 & -8.3514779901625 & 8.7561429689021 \\
\hline $\mathrm{H} 2$ & -2.8303561325086 & -7.8898067051606 & 8.9355637616494 \\
\hline C3 & -3.8244035204650 & -9.3823240435069 & 9.6855506044896 \\
\hline $\mathrm{H} 4$ & -4.7536360666814 & -9.8720818068555 & 9.4294913480836 \\
\hline H5 & -3.0237655255862 & -10.1166091482047 & 9.6448403278832 \\
\hline $\mathrm{H} 6$ & -3.9028034344605 & -9.0006982988925 & 10.7004734513383 \\
\hline \multicolumn{4}{|c|}{ FRAGNAME=WATER } \\
\hline 01 & -3.5803859000660 & -8.9907613392860 & 5.8043435313009 \\
\hline $\mathrm{H} 2$ & -3.6421703452378 & -8.7916588650089 & 6.7248973543548 \\
\hline H3 & -4.0374999147833 & -8.3033002725801 & 5.3468257574603 \\
\hline \multicolumn{4}{|c|}{ FRAGNAME=MEOH } \\
\hline 01 & 3.4451384345068 & -8.3841753765364 & 8.3315872285057 \\
\hline $\mathrm{H} 2$ & 3.9834592968380 & -7.6942825595877 & 7.9822140114622 \\
\hline C3 & 3.3255805815024 & -8.2585084189874 & 9.7220597674686 \\
\hline $\mathrm{H} 4$ & 2.7010217788277 & -9.0727019065046 & 10.0625262142520 \\
\hline H5 & 4.2902391033346 & -8.3318560322181 & 10.2179500822922 \\
\hline $\mathrm{H} 6$ & 2.8541104669152 & -7.3200911440109 & 10.0030261170540 \\
\hline \multicolumn{4}{|c|}{ FRAGNAME=WATER } \\
\hline 01 & -.7994779513362 & -9.1238342172563 & 4.9227073916671 \\
\hline $\mathrm{H} 2$ & -1.6910761815323 & -9.1639542815831 & 5.2298253338608 \\
\hline $\mathrm{H} 3$ & -.5913539169554 & -9.9788974392665 & 4.5814893869453 \\
\hline
\end{tabular}

$\begin{array}{llll}\begin{array}{l}\text { Isomer 2 } \\ \text { FRAGNAME=WATER }\end{array} & & \\ \text { O1 } & -1.3927794238562 & -3.5972278469339 & 6.7526963405814 \\ \text { H2 } & -1.8517520371871 & -4.3070019834032 & 6.3326448324555 \\ \text { H3 } & -.6561195409499 & -3.9790821048592 & 7.2025829892924 \\ \text { FRAGNAME=MEOH } & & \\ \text { O1 } & -3.1725671235775 & -5.2880815587552 & 4.9378632005239 \\ \text { H2 } & -2.7106362731140 & -4.9600065794598 & 4.1850087711446 \\ \text { C3 } & -4.4870141936942 & -4.8029943610353 & 4.9583438380729 \\ \text { H4 } & -4.9630618599734 & -5.2111973377633 & 5.8390543942261 \\ \text { H5 } & -4.5154633654015 & -3.7180578919197 & 5.0212456906531 \\ \text { H6 } & -5.0478931977595 & -5.1211815575512 & 4.0831154157198\end{array}$




\begin{tabular}{|c|c|c|c|}
\hline 01 & -2.8481414508481 & -8.3870860727984 & 4.6951999990702 \\
\hline $\mathrm{H} 2$ & -2.6455760272129 & -7.5168510640544 & 4.9943009405427 \\
\hline C3 & -4.1481948636229 & -8.4381974978606 & 4.1748646339399 \\
\hline $\mathrm{H} 4$ & -4.3211920659862 & -9.4548673767831 & 3.8502780356870 \\
\hline H5 & -4.8928963348387 & -8.1830563899018 & 4.9246485189657 \\
\hline H6 & -4.2665447444567 & -7.7772835968098 & 3.3198562938478 \\
\hline \multicolumn{4}{|c|}{ FRAGNAME=WATER } \\
\hline 01 & -.0725924585881 & -9.1544211564561 & 3.6564883308634 \\
\hline $\mathrm{H} 2$ & .2800589736389 & -9.5478652476248 & 4.4386118304082 \\
\hline H3 & -.9937809990120 & -9.0199727105166 & 3.8120959863422 \\
\hline \multicolumn{4}{|c|}{ FRAGNAME=WATER } \\
\hline 01 & -.7100904519145 & -7.0725013259125 & 6.4671844680869 \\
\hline $\mathrm{H} 2$ & -.1083338455936 & -6.5019033952189 & 6.9179454272492 \\
\hline H3 & -.3260887408801 & -7.9346538883490 & 6.4778661849103 \\
\hline \multicolumn{4}{|c|}{ FRAGNAME=MEOH } \\
\hline 01 & .9772038862733 & -6.4309622613173 & 3.0128760298017 \\
\hline $\mathrm{H} 2$ & .6028096896923 & -7.2269282577659 & 3.3506188499323 \\
\hline C3 & 1.5960064085542 & -6.6639820209795 & 1.7774468023385 \\
\hline $\mathrm{H} 4$ & 2.0039086797293 & -5.7202536952701 & 1.4429401224006 \\
\hline H5 & 2.4084870592453 & -7.3812780181008 & 1.8623866940993 \\
\hline H6 & .8880691876676 & -7.0183147536308 & 1.0323787877400 \\
\hline \multicolumn{4}{|c|}{ FRAGNAME=WATER } \\
\hline 01 & -1.2516692500020 & -4.3082913565975 & 2.8467993328906 \\
\hline $\mathrm{H} 2$ & -.5648772119003 & -4.9534045106338 & 2.9017917774349 \\
\hline $\mathrm{H} 3$ & -.9006154971966 & -3.5073684975902 & 3.2019926473382 \\
\hline \multicolumn{4}{|c|}{ FRAGNAME=MEOH } \\
\hline 01 & 1.1520639942723 & -5.0512435871762 & 7.7378367117413 \\
\hline $\mathrm{H} 2$ & 1.7536740689759 & -5.0150355763417 & 7.0135733670576 \\
\hline C3 & 1.8384726068854 & -5.2894920109710 & 8.9359940683245 \\
\hline $\mathrm{H} 4$ & 1.0999645149498 & -5.3201248442451 & 9.7250248422304 \\
\hline H5 & 2.3641722597943 & -6.2408800140509 & 8.9171822908209 \\
\hline H6 & 2.5490910917655 & -4.4972744479957 & 9.1579531690229 \\
\hline \multicolumn{4}{|c|}{ FRAGNAME=WATER } \\
\hline 01 & 2.6392080644972 & -2.1499694274656 & 4.4106593210108 \\
\hline $\mathrm{H} 2$ & 1.7113611461904 & -1.9772689338118 & 4.4230322651986 \\
\hline H3 & 2.9718741116136 & -1.7876183414143 & 3.6051076804557 \\
\hline \multicolumn{4}{|c|}{ FRAGNAME=MEOH } \\
\hline 01 & -.3949663116637 & -1.8629054682835 & 4.5461279937144 \\
\hline $\mathrm{H} 2$ & -.6828584366317 & -2.3512391687269 & 5.2987576608672 \\
\hline C3 & -1.0470698944796 & -.6241637983073 & 4.4846451636883 \\
\hline $\mathrm{H} 4$ & -.6776748978012 & -.1134528645463 & 3.6062239588752 \\
\hline H5 & -2.1239292824661 & -.7396433800311 & 4.3903505977999 \\
\hline $\mathrm{H} 6$ & -.8322351250829 & -.0116982218415 & 5.3567604542464 \\
\hline \multicolumn{4}{|c|}{ FRAGNAME=WATER } \\
\hline 01 & 2.6888117857151 & -5.0980792740933 & 5.1436523024465 \\
\hline $\mathrm{H} 2$ & 2.8386738375955 & -4.2047361382727 & 4.8784017394764 \\
\hline H3 & 2.1097149196173 & -5.4779584841228 & 4.5023902191057 \\
\hline \multicolumn{4}{|c|}{ FRAGNAME $=\mathrm{MEOH}$} \\
\hline 01 & 4.2108137231721 & -7.6483607102071 & 5.9558422097063 \\
\hline $\mathrm{H} 2$ & 3.9368694383457 & -6.7591031913641 & 5.8075900984975 \\
\hline C3 & 4.0112713349519 & -8.4228273231088 & 4.8052404337435 \\
\hline $\mathrm{H} 4$ & 4.3419677713733 & -9.4260313816970 & 5.0357260477620 \\
\hline H5 & 2.9624409248731 & -8.4609522542680 & 4.5217719152727 \\
\hline $\mathrm{H} 6$ & 4.5915684809387 & -8.0520687167757 & 3.9640232092930 \\
\hline \multicolumn{4}{|c|}{ FRAGNAME=MEOH } \\
\hline 01 & -3.3946103716755 & -7.2955982631935 & 7.9183876656163 \\
\hline $\mathrm{H} 2$ & -2.5807136666511 & -7.1584240406718 & 7.4639006133125 \\
\hline C3 & -3.5876785764452 & -6.3056778876580 & 8.8911581752696 \\
\hline $\mathrm{H} 4$ & -4.5313517998112 & -6.5152493377558 & 9.3753717534613 \\
\hline H5 & -2.8024389754220 & -6.3204689924666 & 9.6428450333757 \\
\hline $\mathrm{H} 6$ & -3.6375404498754 & -5.3126586139321 & 8.4515223531289 \\
\hline \multicolumn{4}{|c|}{ FRAGNAME=WATER } \\
\hline
\end{tabular}




\begin{tabular}{|c|c|c|c|}
\hline O1 & -2.4639685911031 & -10.1732539644927 & 7.2041951925933 \\
\hline $\mathrm{H} 2$ & -2.8366045788677 & -9.6321922362346 & 7.8818920871594 \\
\hline H3 & -2.6146426483123 & -9.7262658867452 & 6.3866516519159 \\
\hline \multicolumn{4}{|c|}{ FRAGNAME=MEOH } \\
\hline 01 & 2.9238198279464 & -9.1736441422823 & 8.2441285518227 \\
\hline $\mathrm{H} 2$ & 3.3214343767279 & -8.6268380131382 & 7.5878444500699 \\
\hline C3 & 3.8771031215196 & -10.0250102767226 & 8.8185211121569 \\
\hline $\mathrm{H} 4$ & 3.3643214693800 & -10.6233446186260 & 9.5587596730459 \\
\hline H5 & 4.3192564314283 & -10.6907529418293 & 8.0815405867430 \\
\hline H6 & 4.6695005379228 & -9.4681724256604 & 9.3123744128693 \\
\hline \multicolumn{4}{|c|}{ FRAGNAME=WATER } \\
\hline 01 & .4591428059060 & -9.8754933853345 & 6.6300283174600 \\
\hline $\mathrm{H} 2$ & -.2994532119256 & -10.2283752490173 & 7.0669321124554 \\
\hline H3 & 1.1553541205156 & -9.8577932237574 & 7.2670957893633 \\
\hline \multicolumn{4}{|c|}{ Isomer 3} \\
\hline \multicolumn{4}{|c|}{ FRAGNAME=WATER } \\
\hline 01 & -1.0690728200736 & -2.5631016830839 & 6.2109103736732 \\
\hline $\mathrm{H} 2$ & -.6078859596278 & -3.1472495950866 & 6.7913897882286 \\
\hline $\mathrm{H} 3$ & -1.9836403964237 & -2.6226249375672 & 6.4365241687576 \\
\hline \multicolumn{4}{|c|}{ FRAGNAME $=M E O H$} \\
\hline 01 & -3.9358038962764 & -4.2473936968432 & 3.6270331183009 \\
\hline $\mathrm{H} 2$ & -3.1182723283661 & -3.9122979765187 & 3.2996860728337 \\
\hline C3 & -3.9009582088597 & -5.6464258300677 & 3.6977085335342 \\
\hline $\mathrm{H} 4$ & -4.8601508084957 & -5.9697478907456 & 4.0775960647614 \\
\hline H5 & -3.7469097792596 & -6.0958174077017 & 2.7198693689803 \\
\hline $\mathrm{H} 6$ & -3.1251973033303 & -5.9967725188623 & 4.3739520958882 \\
\hline \multicolumn{4}{|c|}{ FRAGNAME $=\mathrm{MEOH}$} \\
\hline O1 & -.6056615978685 & -9.3301420132239 & 4.1316165769792 \\
\hline $\mathrm{H} 2$ & -.2864502599922 & -9.5684482928228 & 4.9855002536944 \\
\hline C3 & -1.9718210166704 & -9.6205209275526 & 4.0185295548380 \\
\hline $\mathrm{H} 4$ & -2.2781641204975 & -9.3270740816816 & 3.0240724235925 \\
\hline H5 & -2.1699381070242 & -10.6822598509094 & 4.1422761409923 \\
\hline $\mathrm{H} 6$ & -2.5634765507103 & -9.0639478679965 & 4.7410413533153 \\
\hline \multicolumn{4}{|c|}{ FRAGNAME=WATER } \\
\hline O1 & -4.0647724527773 & -2.9758773417693 & 6.3348500321329 \\
\hline $\mathrm{H} 2$ & -4.6703329261796 & -2.2550530528688 & 6.4025797276239 \\
\hline $\mathrm{H} 3$ & -4.1490391826917 & -3.3196766254235 & 5.4598759611708 \\
\hline \multicolumn{4}{|c|}{ FRAGNAME=WATER } \\
\hline 01 & -.9502470119837 & -6.8371770570126 & 6.2215400194292 \\
\hline $\mathrm{H} 2$ & -.4989298408536 & -6.1324694666796 & 6.6580929683801 \\
\hline $\mathrm{H} 3$ & -.5570935966734 & -7.6399952298390 & 6.5245116806072 \\
\hline \multicolumn{4}{|c|}{ FRAGNAME $=\mathrm{MEOH}$} \\
\hline 01 & .2205956177607 & -6.4754816939718 & 3.3370097486283 \\
\hline $\mathrm{H} 2$ & -.1961804818472 & -7.0939871796558 & 3.9128150468499 \\
\hline C3 & .4379827955334 & -7.0465800650201 & 2.0760221945380 \\
\hline $\mathrm{H} 4$ & .9153574640420 & -6.2939825626825 & 1.4639661841593 \\
\hline H5 & 1.0928547751144 & -7.9124585437360 & 2.1330335171174 \\
\hline $\mathrm{H} 6$ & -.4944301638280 & -7.3392896199751 & 1.5998076103686 \\
\hline \multicolumn{4}{|c|}{ FRAGNAME=WATER } \\
\hline O1 & -.9223137937499 & -3.6570147514070 & 3.3696061338469 \\
\hline $\mathrm{H} 2$ & -.6185827743355 & -4.5501947629677 & 3.3988531655149 \\
\hline H3 & -.9128996091856 & -3.3380520389753 & 4.2578924701568 \\
\hline \multicolumn{4}{|c|}{ FRAGNAME $=\mathrm{MEOH}$} \\
\hline O1 & .7727367128497 & -4.6300298743126 & 7.4389225777234 \\
\hline $\mathrm{H} 2$ & 1.4102841251977 & -4.6710219267769 & 6.7463505286709 \\
\hline C3 & 1.3938769633484 & -4.7639443598005 & 8.6878228322801 \\
\hline $\mathrm{H} 4$ & .6165052646783 & -4.7093182242714 & 9.4372301727320 \\
\hline $\mathrm{H} 5$ & 1.9015353362132 & -5.7203231629601 & 8.7851660486498 \\
\hline H6 & 2.1075966647907 & -3.9646159777188 & 8.8709388225546 \\
\hline \multicolumn{4}{|c|}{ FRAGNAME=WATER } \\
\hline 01 & 1.9880257834310 & -2.3693968071845 & 3.4149802848049 \\
\hline
\end{tabular}




$\begin{array}{llll}\text { H2 } & 1.8489215737043 & -1.6522833578778 & 4.0127066216241 \\ \text { H3 } & 1.1439060784349 & -2.5900902918329 & 3.0549320053499 \\ \text { FRAGNAME=MEOH } & & \\ \text { O1 } & .9199006775842 & -.3301643368497 & 5.4624304299473 \\ \text { H2 } & .2234640670420 & -.8826962967181 & 5.7746604338009 \\ \text { C3 } & .4199998958232 & .9207716056806 & 5.0767380368083 \\ \text { H4 } & 1.2603499930590 & 1.5060672146170 & 4.7301216801045 \\ \text { H5 } & -.2995122863711 & .8350038380742 & 4.2663065158520 \\ \text { H6 } & -.0454475020023 & 1.4429851158589 & 5.9089068358913 \\ \text { FRAGNAME=WATER } & & \\ \text { O1 } & 2.3469839897517 & -4.9738320289918 & 4.9051266247199 \\ \text { H2 } & 2.4652324306071 & -4.1422638407931 & 4.4745571080578 \\ \text { H3 } & 1.7215444417511 & -5.4590060015878 & 4.3910157007164 \\ \text { FRAGNAME=MEOH } & & \\ \text { O1 } & 3.8973302811266 & -7.4579200458330 & 5.7991373140662 \\ \text { H2 } & 3.6585897964293 & -6.5686892474756 & 5.5989507443250 \\ \text { C3 } & 3.5337784318311 & -8.3128691160450 & 4.7501414674289 \\ \text { H4 } & 3.8313496342641 & -9.3120648542238 & 5.0364304661470 \\ \text { H5 } & 2.4601954741311 & -8.3073558160346 & 4.5791374414380 \\ \text { H6 } & 4.0405795807793 & -8.0530230138933 & 3.8241362087269 \\ \text { FRAGNAME=MEOH } & & \\ \text { O1 } & -3.7218996325152 & -7.5861791978979 & 7.1746678662749 \\ \text { H2 } & -2.9230696448150 & -7.1991545459641 & 6.8586209330003 \\ \text { C3 } & -4.4696284965707 & -6.6485274161622 & 7.8993821741997 \\ \text { H4 } & -5.3682475345746 & -7.1481340412009 & 8.2337161079450 \\ \text { H5 } & -3.9290715500825 & -6.2888488300481 & 8.7713240545681 \\ \text { H6 } & -4.7548380767268 & -5.7983828969595 & 7.2847579537371 \\ \text { FRAGNAME=WATER } & & \\ \text { O1 } & -2.5237652987511 & -9.9713142284352 & 8.5807160760834 \\ \text { H2 } & -3.0101771804696 & -9.2783090302019 & 8.1635521627206 \\ \text { H3 } & -3.0093986854744 & -10.7678819677222 & 8.4374732595286 \\ \text { FRAGNAME=MEOH } & & \\ \text { O1 } & 2.6880168765858 & -8.5643115617877 & 8.3352488109483 \\ \text { H2 } & 3.0934073243099 & -8.1608216005037 & 7.5864781314189 \\ \text { C3 } & 3.6237626381544 & -9.3210468565355 & 9.0530452371264 \\ \text { H4 } & 3.1027406789346 & -9.7562093718264 & 9.8945152828177 \\ \text { H5 } & 4.0397237397630 & -10.1247215137242 & 8.4506030840634 \\ \text { H6 } & 4.4362661780999 & -8.7051059119400 & 9.4302824183128 \\ \text { FRAGNAME=WATER } & & \\ \text { O1 } & .0812534607528 & -9.5064144497920 & 7.1404360504049 \\ \text { H2 } & -.5873403538357 & -9.7765656206406 & 7.7494344987205 \\ \text { H3 } & .8643326849186 & -9.3573108651892 & 7.6458403477809\end{array}$

